\title{
Recent Progress in the Interpretation of Stellar Spectra
}

\author{
Otтo Struve \\ Yerkes Observatory, University of Chicago, Williams Bay, Wisconsin, and McDonald Observatory, \\ University of Texas, Fort Davis, Texas
}

\section{PECULIAR STELLAR SPECTRA}

$T$ HE modern interpretation of stellar spectra dates from the appearance, in 1920, of M. N. Saha's classical paper "Ionization in the Solar Chromosphere." ${ }^{1}$ Since that time the theory of ionization and excitation of stellar atmospheres has been developed in considerable detail by H. N. Russell, E. A. Milne, R. H. Fowler, and many others. This theory, which can be designated as the "classical theory" of stellar spectra, is dominated throughout by the concept of thermodynamic equilibrium and the two fundamental equilibrium formulae based upon it: the Boltzmann formula which determines the ratio of the number of atoms in an excited state to the number of atoms in the ground state, and the Saha ionization formula which determines the ratios of the number of ionized atoms to the number of unionized atoms. The particular mechanisms of excitation and ionization-photoelectric ionization, collisions, etc.-play no conspicuous part in this theory and are rarely mentioned in that part of the astrophysical literature of the past quarter of a century which is devoted to stellar spectroscopy. The application of the concept of thermodynamic equilibrium with its corollary, the concept of detailed balancing, is, however, only an approximation. The very fact that spectral lines are formed in a stellar atmosphere proves that the state of the atmosphere cannot be accurately described by means of these concepts. Hence, astrophysicists are led to ask the question how accurate is the "classical theory?" It has been shown that for ordinary stellar atmospheres of stars which resemble the sun, the departures from thermodynamic equilibrium are usually insignificant, and the application of the Boltzmann and Saha formulae is therefore justified. But in recent years, various phenomena have been found which cannot be reconciled with this simplifying idea. Most of them occur in stars which, for one reason or another, have been classified as "peculiar."

${ }^{1}$ M. N. Saha, Phil. Mag. 40, 472 (1920).
Their spectra are unusual, and their atmospheres do not resemble the reversing layer of the sun. The discovery that departures from thermodynamic equilibrium may be important in explaining the spectra of the "peculiar" stars has revived interest in the question of the mechanisms which produce excitation and ionization. This is a field with which physicists-practical and theoretical-are much more familiar than are astronomers. Hence, I propose to discuss several specific problems from this point of view, in the hope that the information now lacking for their complete solution may in time be supplied by the physicists.

In order to understand the peculiar character of the spectra I shall discuss, it is necessary to describe briefly the appearance of a normal stellar spectrum. If we choose all stars included in a typical unit of volume of galactic space, we shall encounter an enormous preponderance-perhaps of the order of ninety-nine percent-of stars, whose spectra consist of absorption lines on the background of a continuous emission spectrum, whose distribution resembles that of a blackbody having a temperature somewhere within the range of $3000^{\circ} \mathrm{K}$ and $100,000^{\circ} \mathrm{K}$. The absorption lines are of atomic, or molecular origin, and have, with few exceptions, been satisfactorily identified. All of these normal stellar' spectra can be classified in a two-dimensional scheme, the relative intensities of the absorption lines changing gradually as functions of two physical parameters: the temperature and the pressure. It is of fundamental importance in astrophysics that the dependence upon temperature is much more pronounced than the dependence upon pressure. Hence, most empirical classification schemes are essentially temperature sequences. The pressure dependence is introduced as a small correction to this principal sequence. For example, among the stars of intermediate temperature, the gradual increase in the intensity of the line $\mathrm{CaI}$ 4226 corresponds to a sequence of decreasing temperature. But two stars having identical lines 
of CaI 4226 may show different intensities for SrII 4215. Because of the pressure dependence of Saha's ionization equation, the star of lower atmospheric pressure shows the stronger line of SrII 4215. This results from the fact that the ionization potential of $\mathrm{Sr}^{+}$is higher than that of $\mathrm{Ca}, \mathrm{Fe}$, and most of the other atoms represented in the spectra of these stars.

An entirely different aspect is presented by the spectra of the nebulae (Fig. 1). Here we see a series of strong emission lines superposed over a continuous spectrum which presents a fairly conspicuous break at $\lambda 3647$, the limit of the Balmer series. The continuum consists of a superposition of a true recombination spectrum of $\mathrm{H}$, and what appears to be a blackbody continuum produced by the non-selective scattering of star light by many small particles in the nebula.

The emission lines in the nebulae are excited by three mechanisms, as was first clearly recognized by Bowen:

(1) The continuous radiation of stars located in, or near, the nebulae causes photoelectric ionization of $\mathrm{H}, \mathrm{He}$, etc., and also produces excitation of atoms through line absorption.

(2) Because of certain chance coincidences in the frequencies of lines excited by the primary mechanism with frequencies of transitions not favored by the primary mechanism, fluorescence plays an important role. Bowen has called attention especially to the fact that certain members of high level multiplets of OIII are strong, while other members are absent. This he attributes to the fact that the line HeII 303.78 $\left(1 s^{2} S-2 p^{2} P\right)$ is undoubtedly exceedingly strong in all nebulae (although atmospheric absorption renders it unobservable) and agrees very closely with the line OIII $303.80\left(2 p^{3} P_{2}-3 d^{3} P_{2}\right)$. The emission of HeII causes the OIII level $3 d^{3} P_{2}$ to be overpopulated, and all lines originating from it are therefore greatly enhanced.

(3) Because high level transitions of certain ions, OIII, OII, NII, etc., are weak in the nebulae, Bowen has suggested that the excitation of low metastable levels occurs predominately by the mechanism of collisions with free electrons. It can be shown that the forbidden lines originating from these levels are too strong to be attributed wholly to the mechanism of recombination.

There exist a number of stars whose spectra combine the normal features of a stellar spectrum with features usually associated with gaseous nebulae. These stars are usually designated as emission-line stars, and the spectroscopic symbols

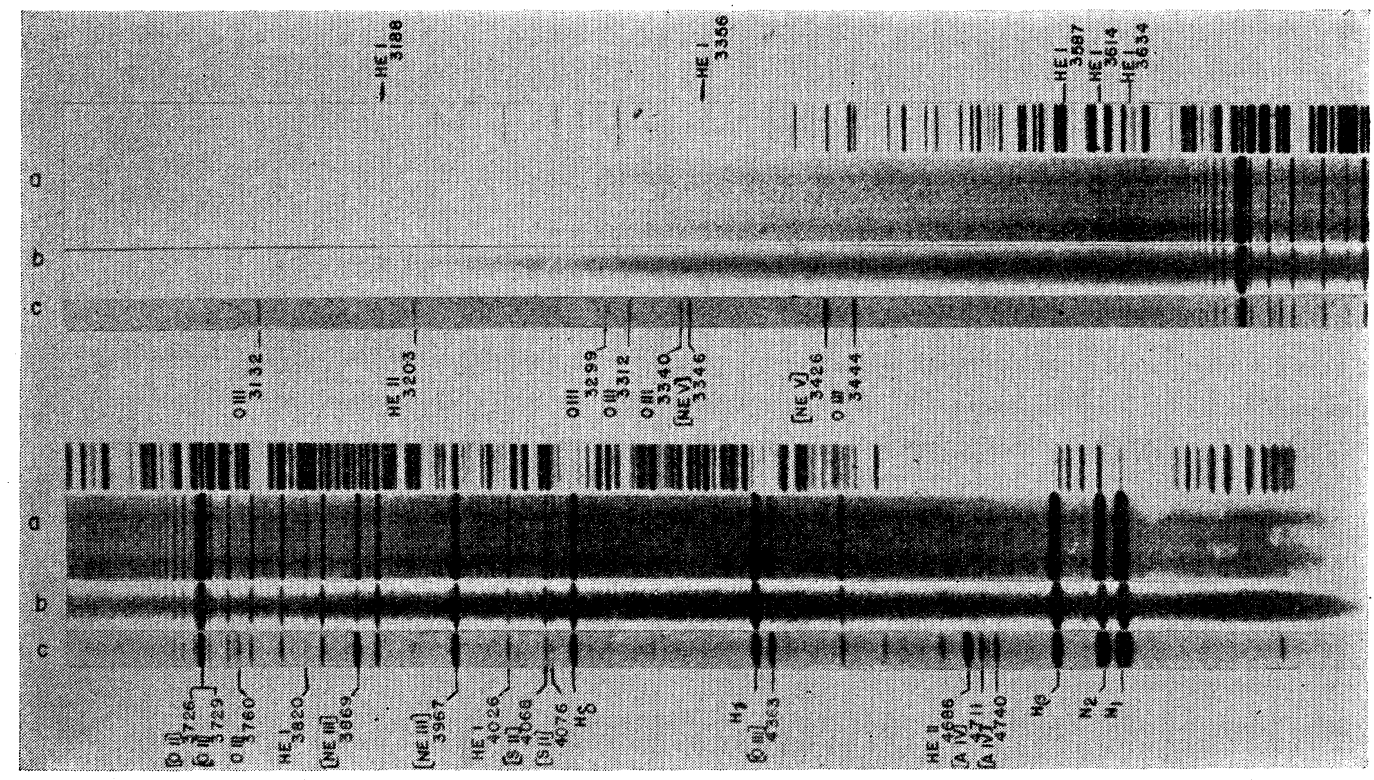

Fig. 1. Nebulae: (a) Orion, (b) IC 418, (c) IC 2165. 
TABLE I. Physical characteristics of stars.

\begin{tabular}{|c|c|c|c|c|c|}
\hline Property Group & White dwarf & Dwarf & Giant & Super-giant & Nebula \\
\hline Example & Sirius B & Sun $=\odot$ & Capella & Betelgeuse & Orion \\
\hline Mass & $1 \odot$ & $1 \odot$ & $5 \odot$ & $15 \odot$ & \\
\hline Radius & $0.01 \odot$ & $1 \odot=700,000 \mathrm{~km}$ & $10 \odot$ & $300 \odot$ & \\
\hline Mean density & $10^{5} \mathrm{~g} / \mathrm{cm}^{3}$ & $1 \mathrm{~g} / \mathrm{cm}^{3}$ & $10^{-3} \mathrm{~g} / \mathrm{cm}^{3}$ & $10^{-6} \mathrm{~g} / \mathrm{cm}^{3}$ & \\
\hline Height of abs. atm. & $100 \mathrm{~km} ?$ & $100 \mathrm{~km}$ & $1000 \mathrm{~km}$ & $100000 \mathrm{~km}$ & $10^{13} \mathrm{~km}$ \\
\hline $\begin{array}{l}\text { Mean electron pressure } \\
\text { of atm. }\end{array}$ & $10^{4}-10^{5} \mathrm{~d} / \mathrm{cm}^{2}$ & $100 \mathrm{~d} / \mathrm{cm}^{2}$ & $1-10 \mathrm{~d} / \mathrm{cm}^{2}$ & $10^{-1} \mathrm{~d} / \mathrm{cm}^{2}$ & \\
\hline $\begin{array}{l}\text { No. of electr. per } \mathrm{cm}^{3} \\
\text { of } \mathrm{atm} \text {. }\end{array}$ & $10^{16}-10^{17}$ & $10^{14}$ & & & $y^{4}$ \\
\hline$T$ & $\sim 10,000^{\circ}$ & 3,000 to $50,000^{\circ}$ & same & same & $50,000^{\circ} \mathrm{K}$ \\
\hline
\end{tabular}

which define their place in the classification scheme are followed by the letter $e$. These stars are quite rare, and most of them are faint. They have been known for many years from the objective prism work of the Harvard Observatory, but in recent years they have been investigated mostly at Mount Wilson by P. W. Merrill, at the University of Michigan by D. B. McLaughlin, at the Victoria Observatory by C. S. Beals, and at the McDonald Observatory by P. Swings, and the writer.

To understand the significance of the emissionline stars, it may be useful to review the principal physical quantities which serve to describe different kinds of stars and nebulae. This has been done in Table I. The data given are only rough approximations for each group and are not the accurate values derived for the stars listed in the first line.

The "classical theory" of stellar atmospheres is concerned with the reversing layers whose properties are described in the last four lines of Table I. But we know that even in a normal star like the sun, the reversing layer is not sharply limited at the top and at the bottom. At the top, it gradually merges into a very rarefied layer, the chromosphere, and this, in turn, gradually merges into the solar corona. The spectrum of the corona consists of a strong continuum in which the absorption features of the normal solar spectrum can be distinguished, probably as a result of non-selective scattering and of a large number of emission lines. The latter have recently been identified ${ }^{2}$ by $B$. Edlén as for-

\footnotetext{
${ }^{2}$ In fairness to the reader not acquainted with the astronomical literature, it should be stated that while these identifications are distinctly probable, they have not yet been established with the same degree of certainty as
}

bidden transitions of $[\mathrm{Fe}] \mathrm{X}, \mathrm{XI}, \mathrm{XIII}, \mathrm{XIV}$, $\mathrm{XV}$; [Ni] XII, XIII, XV, XVI; [Ca] XII, $\mathrm{XIII}, \mathrm{XV}$; [A] X, XIV. The ionization potentials of these ions are as high as 814 volts (for Ca XIV) and represent the highest stages of ionization thus far observed in any astronomical source. The ionization level of the ordinary solar reversing layer is, by contrast, only about 8 volts. The ionization equation shows that ordinary thermal ionization, with the solar temperature, cannot account for this high ionization level in the corona. ${ }^{3}$ Hence, the mechanism of ionization must be something different. Moreover, the existence of a layer showing strong forbidden lines in the vicinity of the sun shows that the concept of thermodynamic equilibrium can certainly not be used in describing its spectrum.

It is of interest to note that Adams and Joy at Mount Wilson have found the strongest coronal lines $\lambda \lambda 5303,6375$ of $[\mathrm{Fe} \mathrm{XIV}]$ and $[\mathrm{Fe} \mathrm{X}]$ in the spectrum of the peculiar star RS Ophiuchi, while Swings and the writer found some evidence of the $[\mathrm{Fe} \mathrm{X}]$ line in another star, $A X$ Persei. ${ }^{3 a}$ The absence of these lines in the integrated spectrum of the sun is not due to

have other identifications in the sun and stars. The reader is referred to Edlén's original papers and to a detailed review by Swings (Ap. J. 98, 116 (1943).

${ }^{3}$ Saha's equation gives $\log \left(n_{r+1} / n_{r}\right) p_{e}=-\chi_{r}(5040 / T)$ $+5 / 2 \log T+$ const. If we consider two such ratios, each giving $n_{r-1} / n_{r}=1$, the one corresponding to $\chi_{r}=8$ volts,

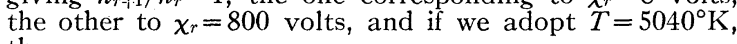
then

$$
\log \left(p_{e} / p_{e}{ }^{\prime}\right)=792,
$$

which would correspond to an impossibly low pressure in the corona-a pressure which would be much lower than that of the interstellar medium and would leave virtually no gas to radiate!

$3 a$ In April, 1945, Joy announced the discovery of the coronal lines in the spectrum of another peculiar star, $T$ Pyxidis. 
any important intrinsic causes; if the corona were much larger than it actually is, the emission lines might be observed in the integrated light, and the sun would then be classified as a peculiar star. It is evident that under normal conditions of development, single stars do not develop such extensive coronas, and the problem is to discover those conditions which render a corona sufficiently large in such stars as $R S$ Ophiuchi and $A X$ Persei.

The emission-line stars are, however, not all as spectacular as $R S$ Ophiuchi and $A X$ Persei. They can be roughly classified into five distinct groups.

A. Ordinary Be stars have normal spectra corresponding to temperatures of the order of $20000^{\circ} \mathrm{K}$ with absorption lines of $\mathrm{H}, \mathrm{HeI}, \mathrm{OII}$, $\mathrm{MgII}$, etc. upon which are superposed emission lines of $\mathrm{H}, \mathrm{FeII}$, and occasionally, of other elements. It has been shown, mostly by the writer, that these emission lines occur predominantly in normal stars whose axial rotation is very rapid - of the order of $300 \mathrm{~km} / \mathrm{sec}$. at the equator-and the suggestion was made that rotational instability causes these stars to dissipate material in the form of rings in their equatorial planes. When such rings are observed projected upon the star-disks (that is, when the line of sight is in the equatorial plane), peculiar absorption lines are formed; the $\mathrm{H}$-lines are exceedingly sharp, being devoid of broadening by Stark effect or by collisions, and those lines (SiII, MgII) whose lower levels are not metastable are greatly weakened, showing that the mechanism of their excitation is to be identified with the dilute continuous radiation of the rotating star. Usually, the ionization level of the shell is lower than that of the normal reversing layers. When lines of FeII are observed, they are almost always those which correspond to permitted transitions. Only in very few Be stars have forbidden $[\mathrm{FeII}]$ lines been observed. An example of this latter group is the star $M W C 56$ (Fig. 2). The diameters of the ring-like structures are estimated to be of the order of two to five times the diameters of their central stars. Particularly interesting and convincing information has been obtained in the cases of several Be or Ae stars which happen to be members of close binary systems. In several such binaries, the lobes of the rings are successively eclipsed by the other components, with an eclipse of the central star itself taking place between.

B. The Wolf-Rayet stars and P-Cygni type stars, for reasons which are not yet understood, are surrounded by rapidly expanding gaseous shells which produce very broad emission lines flanked on their violet sides by absorption lines of somewhat smaller widths. The velocities of expansion range from a few tens of $\mathrm{km} / \mathrm{sec}$. to several thousands of $\mathrm{km} / \mathrm{sec}$. They are not the same for all lines, and definite indications of stratification have been found in the expanding shells. The emission lines correspond to high stages of ionization ( $\mathrm{NV}$, etc.) and are all of permitted transitions. A surprising number of these stars are members of close binary systems, having periods of from 1.6 to more than 20 days. The velocities of the Wolf-Rayet stars in their orbits are measured in hundreds of $\mathrm{km} / \mathrm{sec}$. and

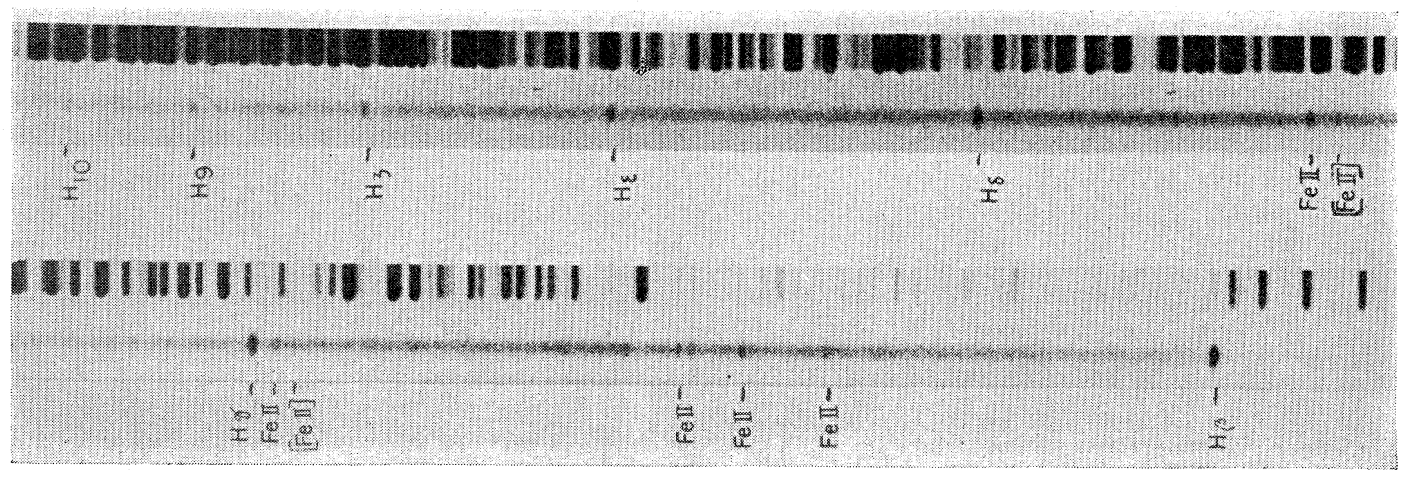

FIG. 2. $M W C 56$. 


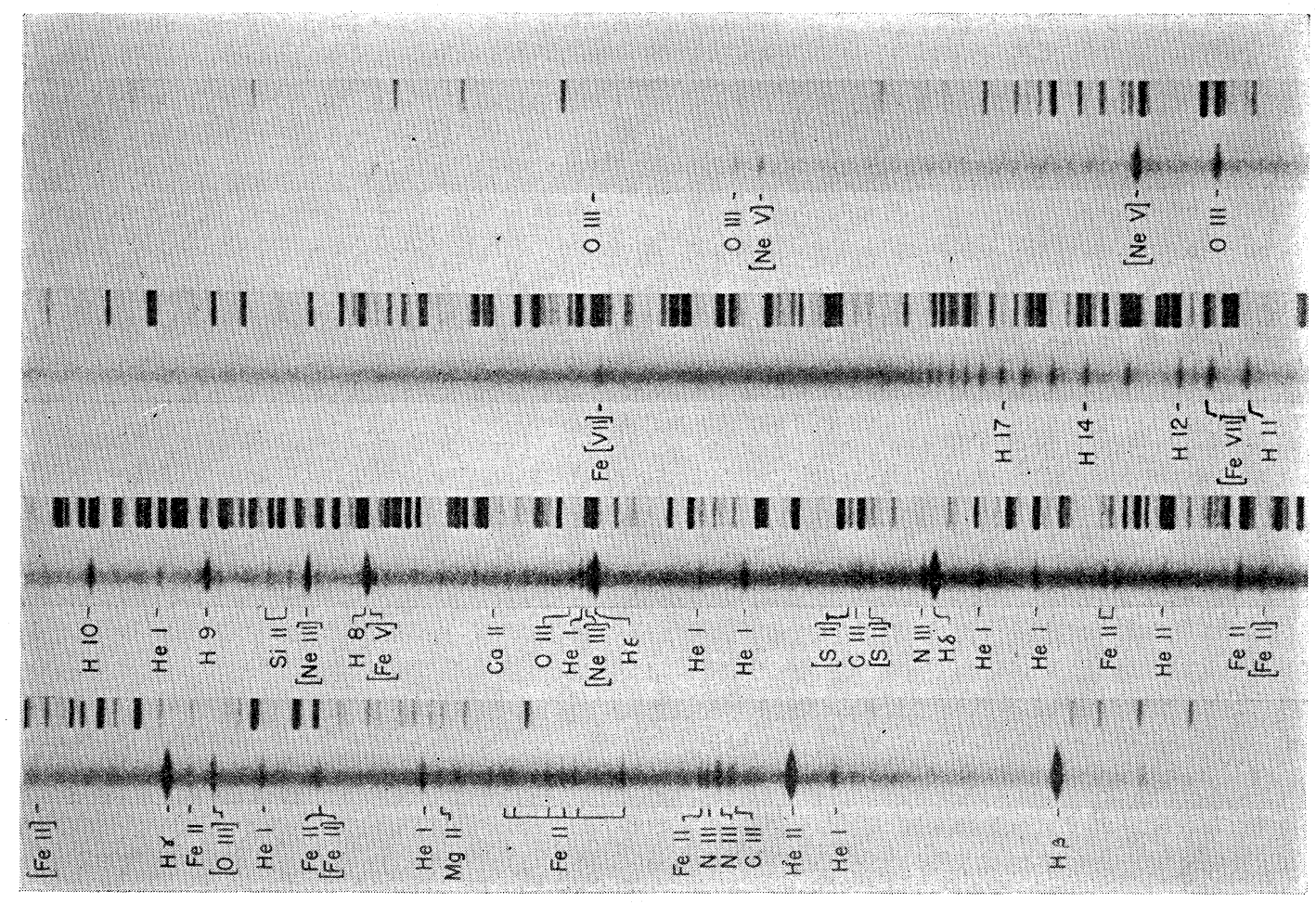

FIG. 3. $Z$ Andromedae.

are much smaller than their velocities of expansion. The emission and violet absorption lines of $P$ Cygni (and of other related objects) show remarkable departures from the laboratory intensities, suggesting that the various levels are populated by highly selective mechanisms and not by a process (such as recombination, or thermal excitation) which would permit the use of the Boltzmann formula.

C. Novae are in many respects similar to stars of the preceding group, but while a Wolf-Rayet star or a $P$-Cygni type star is a fairly stable object (their spectra remain the same over periods of many years), novae, supernovae, and recurrent novae represent cataclysmic phenomena which can best be described by the word explosion.

D. Certain variable stars of long period and low surface temperature develop periodically emission lines of H, FeII, MgI, SiI, and other elements. These stars have been investigated mostly at the Mount Wilson Observatory. Of particular interest is the weakness of the line $\mathrm{H} \epsilon$ which is undoubtedly caused by heavy absorp- tion in overlying gases of CaII. There are other similar cases of absorption lines which originate at higher levels than the emission lines and tend to weaken the latter.

E. Finally, there are a number of peculiar stars which cannot be classed in any of the preceding four groups. The stars $R S$ Ophiuchi and $A X$ Persei are examples. There is no evidence that all these stars are physically related, and several small sub-groups of closely similar objects can already be distinguished. An attempt to isolate these sub-groups and describe them has recently been made by Merrill. ${ }^{4}$ The problems 1 want to discuss concern this group of peculiar stars.

A particularly interesting representative of this group is the variable star $Z$ Andromedae. ${ }^{5}$

${ }^{4}$ Astrophys. J. 99, 22 (1944).

${ }^{5}$ Non-astronomical readers are of ten confused by the bewildering names of individual stars. Greek letters and proper names are used only for the brightest stars. Faint stars may be designated by the numbers which the stars carry in some well-known catalogue. Variable stars are designated by capital letters of the Latin alphabet, but occasionally, the same star may be designated in several different ways. 


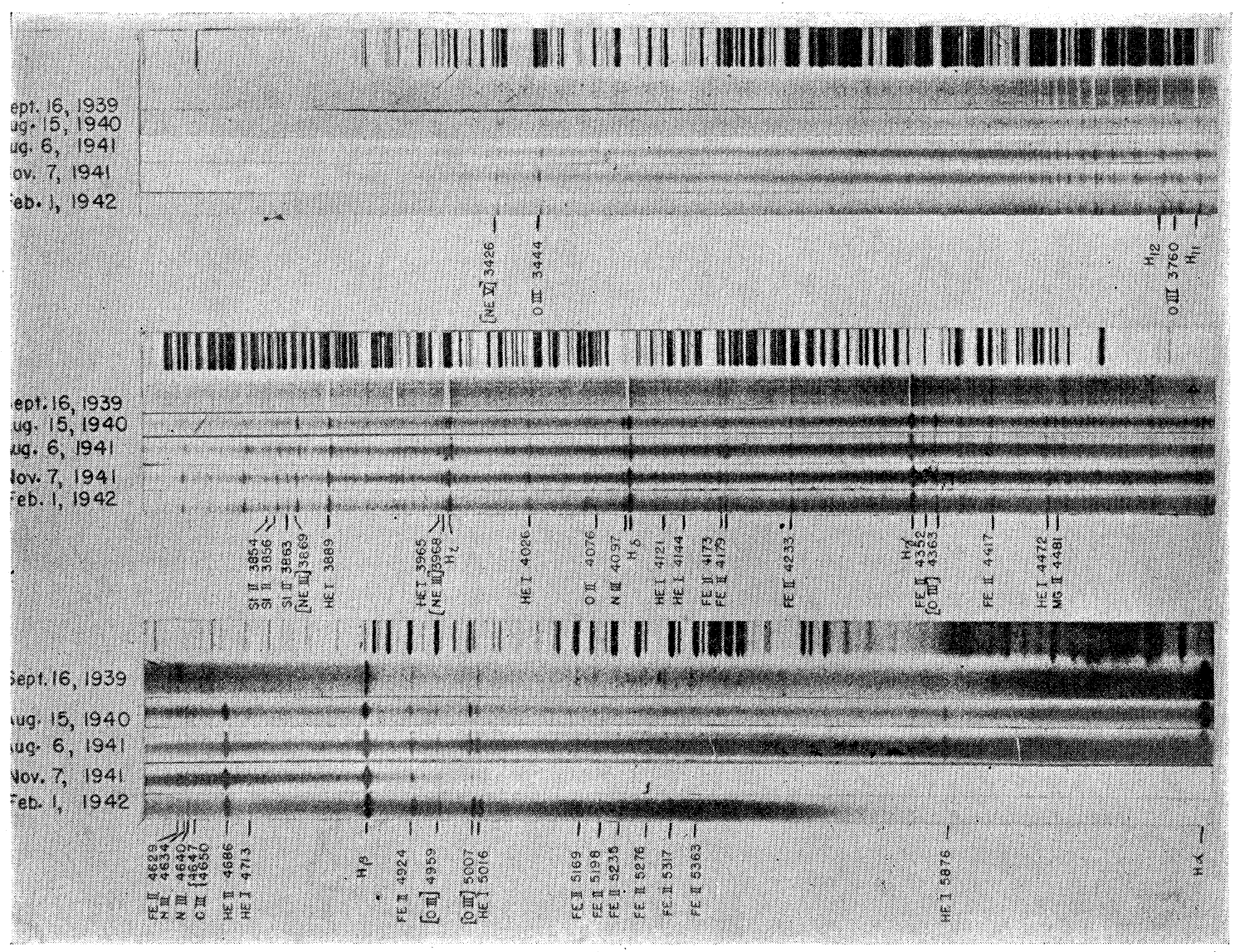

FIg. 4. Changes in the spectrum of $Z$ Andromedae.

Figure 3 shows a spectrogram obtained at the McDonald Observatory in the fall of 1944. The continuous spectrum shows a pronounced "jump" at the Balmer limit. To the violet of $\lambda 3647$, the $\mathrm{H}$ continuum is strong. To the red, the continuum is probably mostly that of a stellar photosphere and not so much that produced by the Paschen continuum. The emission lines show an extraordinary range in ionization potential. Thus, we observe. [FeII] and [FeVII]. A gas at uniform pressure and temperature would not produce such strong lines of ions having such widely different ionization potentials. We conclude that the observed spectrum originates from the integration of spectra of different sources whose ionizations are not the same.

The spectrum of, $Z$ Andromedae undergoes gradual changes. This is shown in Fig. 4. In the fall of 1939 , there was observed a sudden out- burst of light - a phenomenon resembling on a very small scale the outburst of a nova. At this time, the spectrum was that of an expanding shell, with strong absorption lines shifted to the violet side, which were obviously produced in those layers of the expanding atmosphere which were projected upon the apparent disk of the star. ${ }^{6}$ In addition to occasional outbursts at irregular intervals $Z$ Andromedae shows slow fluctuations in the relative intensities of the emission lines (Fig. 5). For example, at certain times the line $[\mathrm{NeV}] 3426$ is much stronger than the fluorescence line OIII 3444, while at other times, the opposite is true. These fluctuations have a period of approximately two years, and they are associated with gradual changes in the

\footnotetext{
${ }^{6}$ It should be remembered that the star is so far away that shell and star could not be seen separately in the telescope and appeared as a single point source.
} 


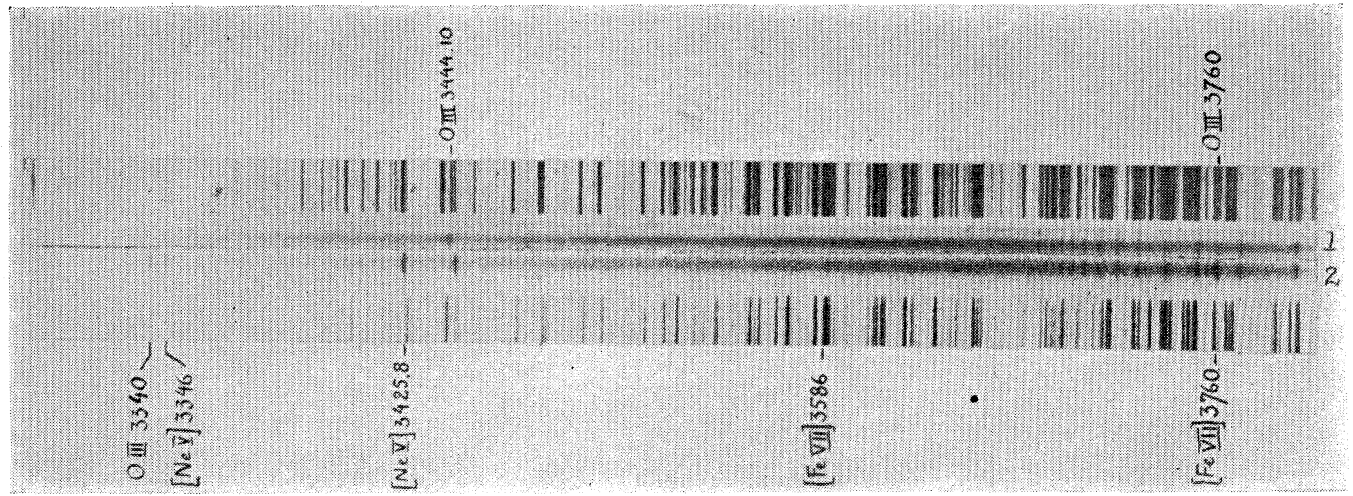

Fig. 5. $Z$ Andromedae.

radial velocities of the different groups of emission lines. Some years ago it was discovered by Merrill that the continuous spectrum of $Z$ Andromedae in the green and yellow regions is broken up by absorption bands of TiO. Such bands occur only in stars of very low surface temperature (about $3000^{\circ} \mathrm{K}$ ), and it was, at first, difficult to understand how a star of such low surface temperature could produce emission lines of $[\mathrm{NeV}],[\mathrm{FeVII}]$, etc., which require large energies of ionization, and of OIII, HeI, HeII, etc., which require also large energies of excitation. Previously, it had always been found that only the hottest stars, having temperatures of the order of 50,000 to $100,000^{\circ} \mathrm{K}$, could produce such emission lines. In fact, Zanstra

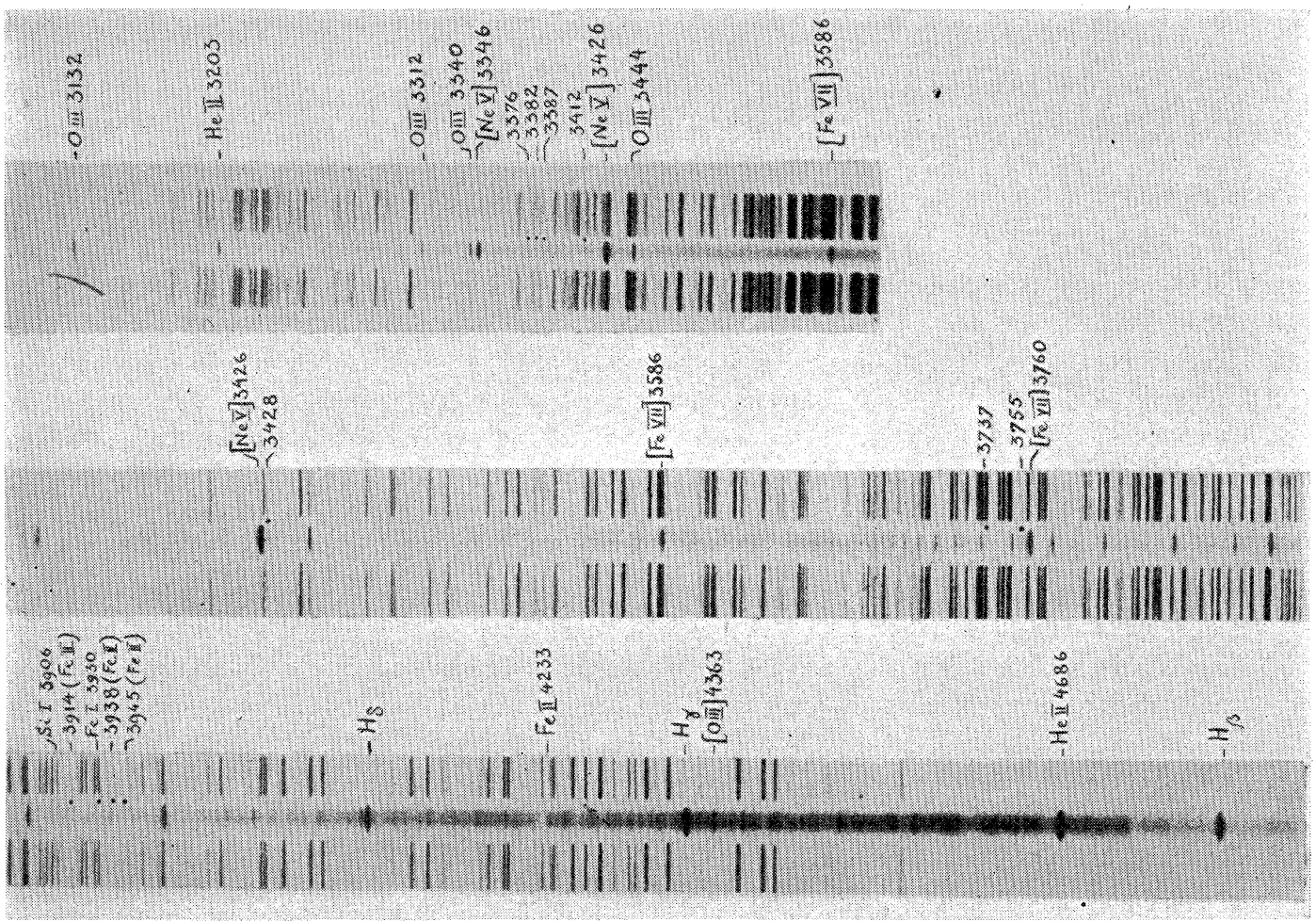

Fig. 6. CI Cygni. 
had developed a theory by means of which it was possible to determine the temperature of the exciting star from measurements of total energies contained in the emission lines. An application of this theory would give for $Z$ Andromedae a temperature of perhaps $50,000^{\circ} \mathrm{K}$.

At the present time, the $\mathrm{TiO}$ absorption bands are not seen in the spectrum of $Z$ Andromedae. But Fig. 6 shows the spectrum of a very similar object, CI Cygni, in which they can be easily distinguished. It is of considerable interest that in $Z$ Andromedae we observe in the same spectrum lines of $[\mathrm{FeII}],[\mathrm{FeV}]$, and $[\mathrm{FeVII}]$, while $[\mathrm{FeIII}]$ is not seen. This may be caused by the accidental absence in the object of a layer in which $[\mathrm{FeIII}]$ is favored. Another star of this group, $B F$ Cygni, shows [FeIII] conspicuously (Fig. 7, taken from a recent paper by P. W. Merrill), so that its absence in $Z$ Andromedae can probably not be attributed to any intrinsic weakness of the lines of [FeIII]. The evidence in regard to $Z$ Andromedae suggests the existence of four separate sources: a cool star producing TiO absorption, a very hot, but presumably exceedingly small, star whose existence is inferred by the presence of lines requiring large excitation energies, a tenuous mass of gasproducing emission lines of relatively low ionization ([FeII $]$ ), and another mass of gas producing lines of high ionization ([FeVII]). In evaluating this picture, we are perhaps somewhat uncertain about the hot star, because we do not observe it directly. Perhaps the source of excitation of the emission lines must be sought in some other type of mechanism than blackbody radiation. The disturbing aspect is the absence of a continuous spectrum corresponding to a high temperature. This is, however, not necessarily fatal. We observe, for example, many planetary nebulae with strong emission lines and with very faint, hot central stars. It requires only a slight effort of the imagination to think of a central star so faint that it would not be observed at all. But are the circumstances really alike? The star $Z$ Andromedae is roughly of the tenth visual magnitude. Most of this light is, however, concentrated in the emission lines and in the cool continuum. The photographic magnitude of the hypothetical hot companion cannot be greater than about 11 . On the other hand, the star cannot be very far from us, because we fail to observe any interstellar lines of CaII. From the known relation between interstellar line intensity and distance, we infer that probably the distance is less than 400 parsecs. At this distance, a star of apparent magnitude 11 would have an absolute magnitude of about +3 . The known hot stars have absolute brightnesses of the order of 1000 times greater. The hot companion, if it exists, must be an underluminous object. Such underluminous stars are not unknown. But the problem of $Z$ Andromedae would then be even more complicated and interesting. Since the temperature of the exciting

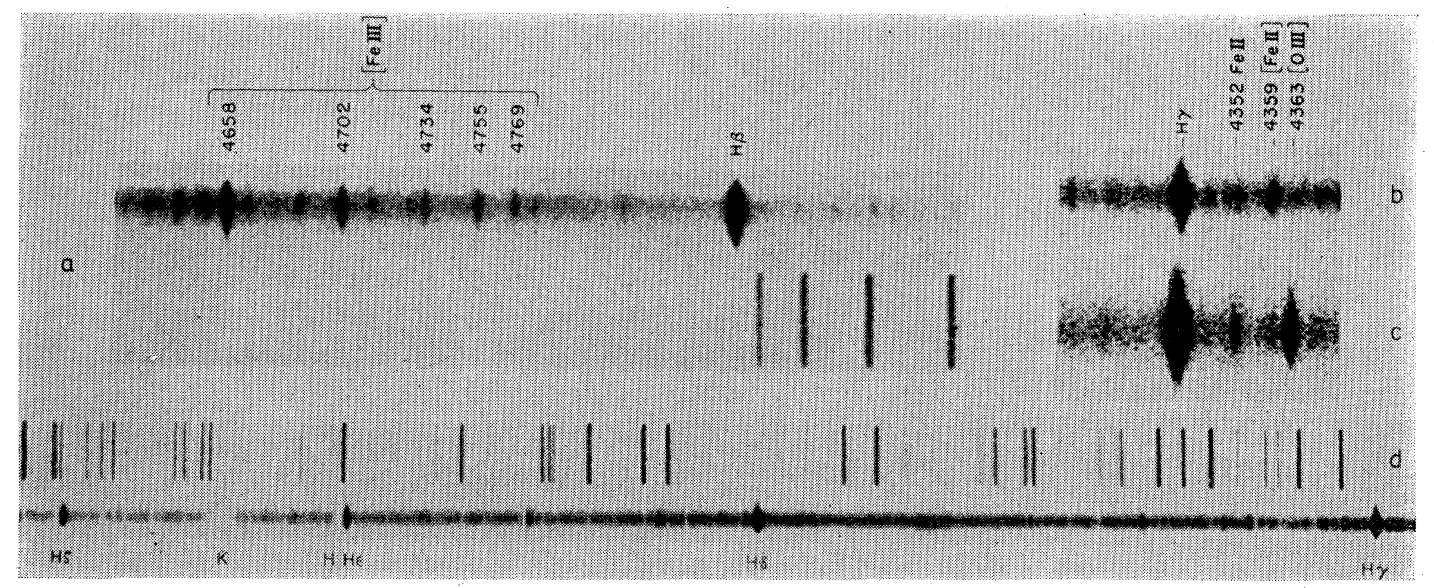

FIg. 7. BF Cygni (a) August 31, 1941; (b) August 6, 1941; (c) September 15, 1942; (d) August 11, 1943. 


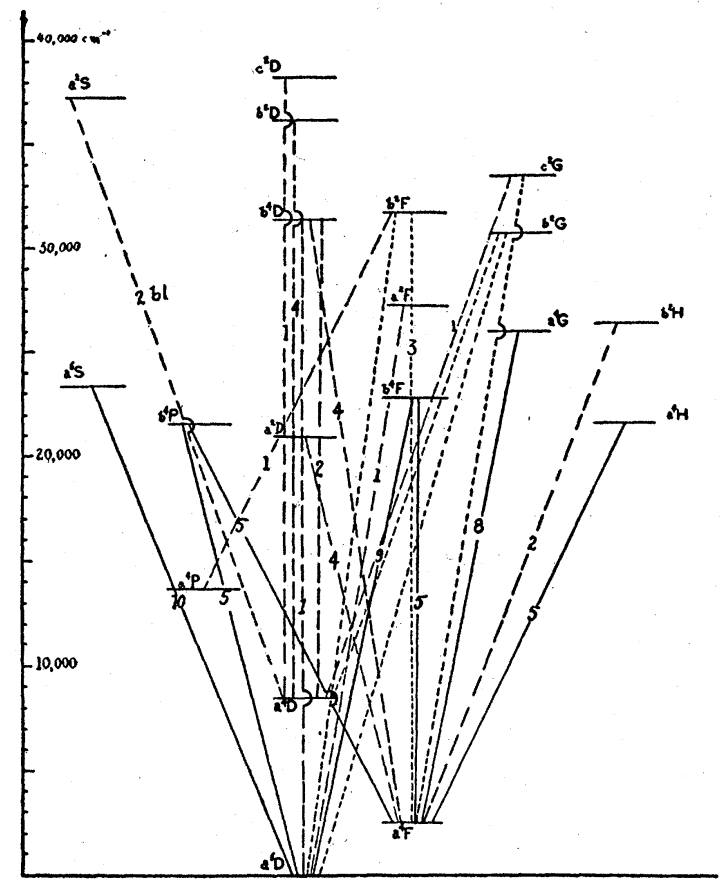

FIG. 8. Forbidden lines of FeII. Full-drawn lines: Forbidden transitions observed in $\eta$ Carinae; dashed lines: new observed lines; dotted lines: transitions probably present.

star must be high, of the order of $50,000^{\circ} \mathrm{K}$, the only reason for the star to be underluminous must be small size. Knowing the total luminosity from the absolute magnitude and the surface brightness from the temperature, we find that the radius of the star is of the order of onethird of that of the sun. This is abnormally small, since a normal star of the same temperature has a radius of five to ten times larger than the sun.

\section{THE PROBLEM OF FeII}

A few months after Bowen identified the "nebulium" lines as forbidden transitions of [OIII], Merrill announced that several strong emission lines in the spectrum of $\eta$ Carinae are caused by forbidden transitions of [FeII]. Since that time many new forbidden transitions of [FeII] have been identified in the spectra of several stars, mostly by P. Swings and the writer. Figure 8 shows the metastable levels of $\mathrm{Fe}^{+}$and gives the forbidden multiplets known about two years ago. A few additional transitions have been identified since then, but the picture, as shown, is essentially complete.

We have already seen that in the spectra of $M W C 56$ and $Z$ Andromedae permitted and forbidden lines occur simultaneously. In $Z$ Andromedae and probably in other stars, the relative intensities $\mathrm{FeII} /[\mathrm{FeII}]$ undergo large changes with time, but on all our spectrograms, the permitted lines are somewhat stronger than the forbidden lines. Figures 9 and 10 , on the contrary, show two stars, Boss 1985 and $W Y$ Geminorum, in whose spectra the forbidden lines of [FeII] are strong while the permitted lines are either absent or exceedingly weak. The most conspicuous forbidden lines of $[\mathrm{FeII}]$ are $\lambda \lambda 4244$, 4277,4287 , and 4359 . We are confronted here with a most remarkable problem, why are the permitted lines suppressed? To answer this question, let us examine the two spectra critically and list their principal characteristics.

1. Both stars have continuous spectra of low temperature and absorption features consisting of TiO, CaI, and FeI which occur only in stars of low temperature, of the order of $3000^{\circ}$ to $4000^{\circ} \mathrm{K}$.

2. Both stars have long ultraviolet extensions in the continuous spectra, with Stark-broadened higher members of the Balmer series in absorption. The spectra are composite and result from the combination of a cool star and a hot star. The temperature of the latter may be of the order of $15,000^{\circ} \mathrm{K}$.

3. There are weak central emission components of $\mathrm{H}$ in Boss 1985, but not in $W Y$ Geminorum. The exciting mechanism is evidently strong enough to excite the forbidden lines of [FeII] in a tenuous mass of gas which must be associated with the binary system, but is barely sufficient to excite $\mathrm{H}$, despite the enormous cosmic abundance of the latter.

After having ascertained these facts, Swings and the writer obtained several spectrograms of both stars in the ultraviolet region. ${ }^{7}$ The purpose of this work was to identify new forbidden lines. To our surprise, we found the spectra full of permitted lines, in emission, of FeII, CrII, etc.,

${ }^{7}$ At $\lambda 3000$, the absorption of the atmosphere causes all astronomical spectra to be sharply cut off. 


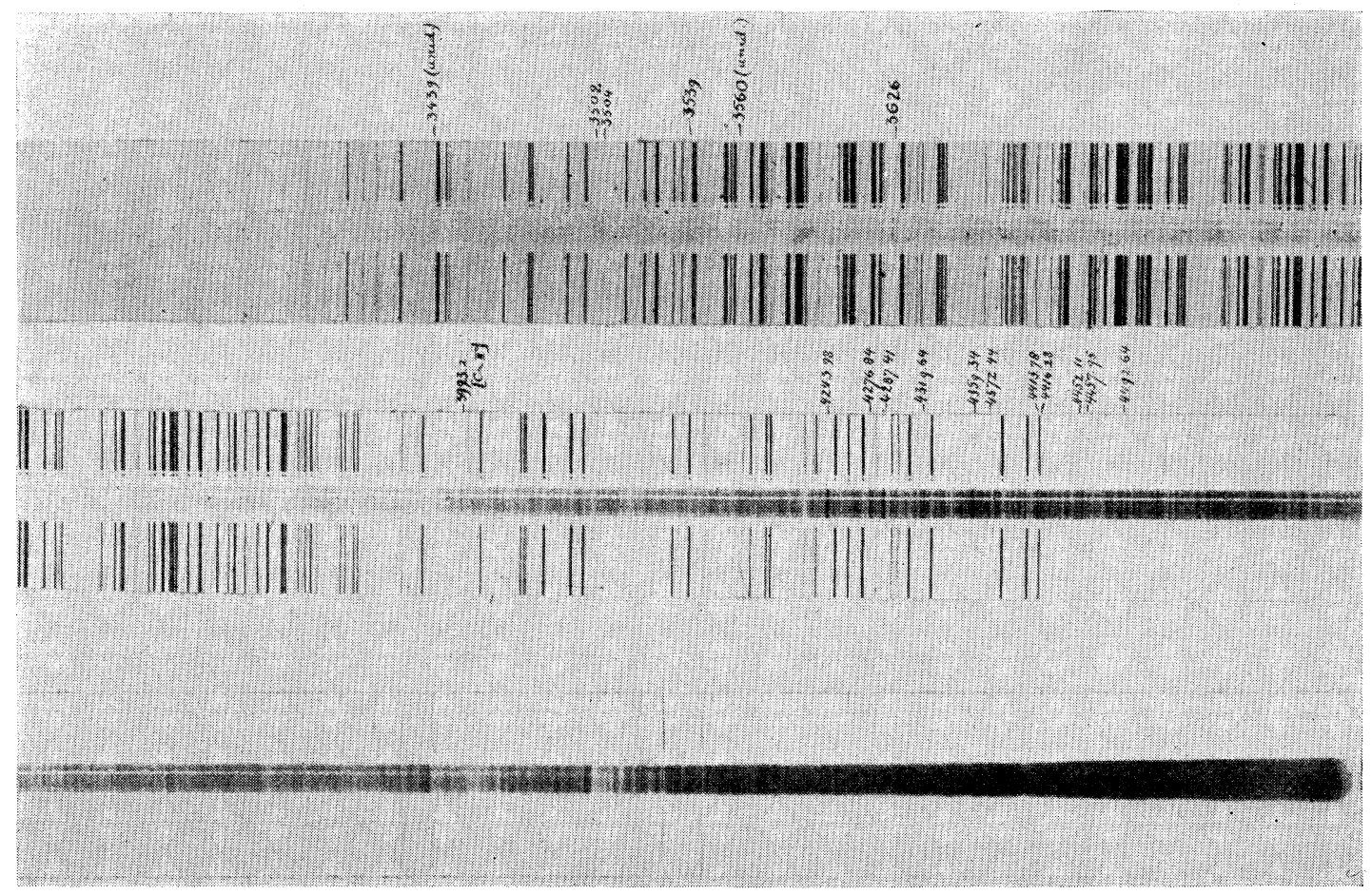

FIG. 9. Boss 1985.

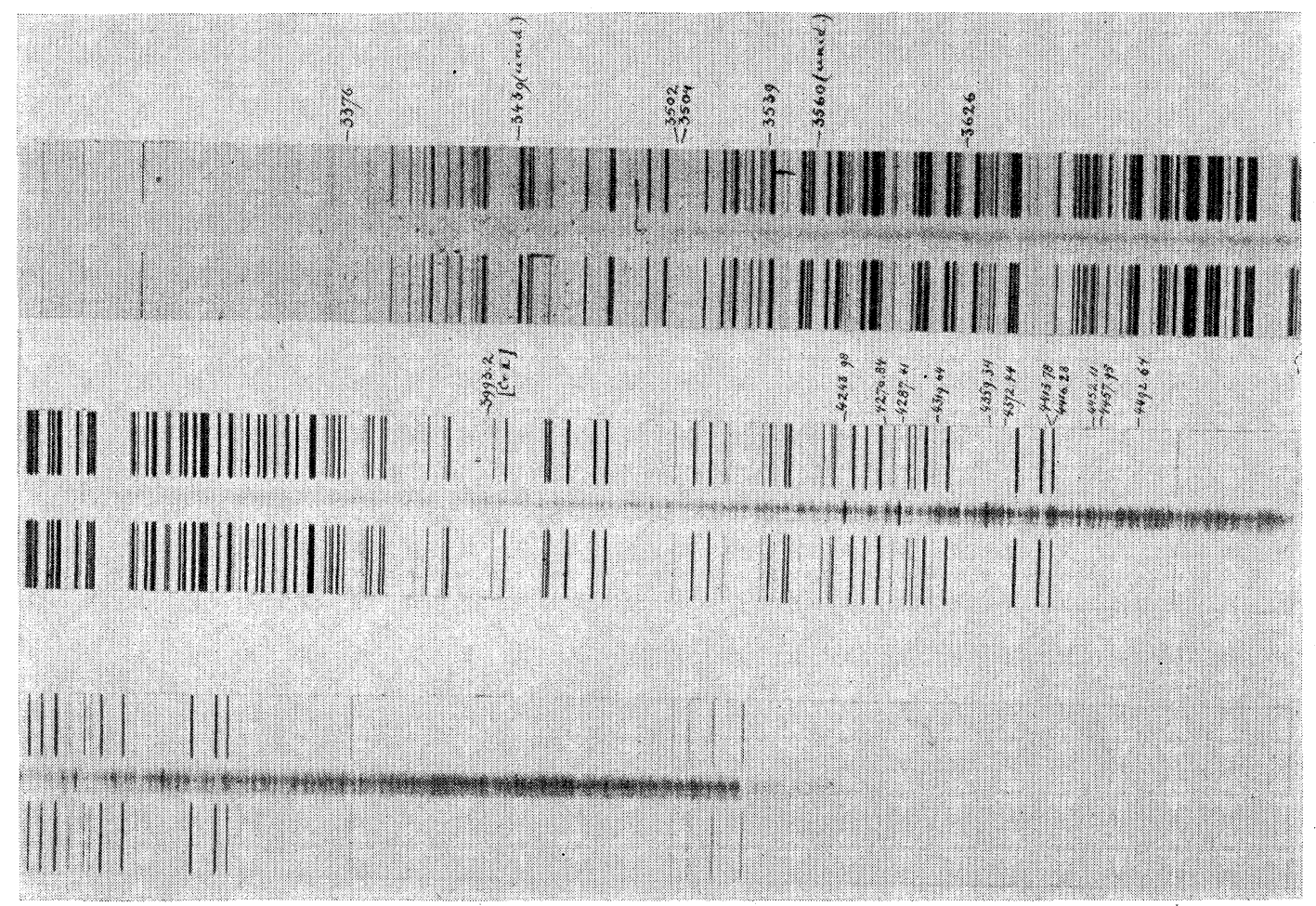

FIG. 10. $W Y$ Geminorum. 
TABLE II. Estimated intensities of FeII lines.

\begin{tabular}{|c|c|c|c|c|c|}
\hline Transition & & $\lambda$ & $\begin{array}{l}\text { Lab. } \\
\text { int. }\end{array}$ & $\begin{array}{l}\alpha \text { Cyg } \\
\text { abs. }\end{array}$ & $\begin{array}{c}\text { Pec. stars } \\
\text { emission }\end{array}$ \\
\hline $\begin{array}{l}b^{4} D-y^{4} F^{0} \\
\text { e.p. } 3.9-7.7 \mathrm{v}\end{array}$ & $\begin{array}{l}3 \frac{1}{2}-4 \frac{1}{2} \\
2 \frac{1}{2}-3 \frac{1}{2}\end{array}$ & $\begin{array}{l}3259 \\
3259\end{array}$ & $\left.\begin{array}{l}10 \\
10\end{array}\right\}$ & 7 & $0-1$ \\
\hline $\begin{array}{l}a^{4} P-z^{4} F^{0} \\
\text { e.p. } 1.7-5.5 \mathrm{v}\end{array}$ & $2 \frac{1}{2}-3 \frac{1}{2}$ & 3196 & 10 & 3 & 5 \\
\hline $\begin{array}{l}d^{2} D-x^{4} F^{0} \\
\text { e.p. } 5.9-9.0 \mathrm{v}\end{array}$ & $1 \frac{1}{2}-2 \frac{1}{2}$ & 3939 & 4 & 4 & 0 \\
\hline $\begin{array}{l}a^{4} P-z^{6} D^{0} \\
\text { e.p. } 1.7-4.8 \mathrm{v}\end{array}$ & $2 \frac{1}{2}-2 \frac{1}{2}$ & 3938 & 2 & 6 & 3 \\
\hline
\end{tabular}

together with all the expected forbidden lines. Upon investigation it turned out that the permitted lines of FeII were all low level transitions: The observed stellar intensities were quite abnormal. Those corresponding to low levels were greatly enhanced as compared with the usual laboratory intensities or with the absorption intensities of normal stellar spectra. This at once explained the great intensity of [FeII]. These lines were not favored by some exceptional mechanism, but were enhanced because their excitation levels are lower than those of the permitted FeII lines. A few examples will illustrate this point. (See Table II.)

We can define an excitation temperature $T^{\prime}$ by making use of the Boltzmann formula for two states $s$ (e.p. $E_{s}=2.5$ volts) and $t$ (e.p. $E_{t}=5.5$ volts) and in two stars, for one of which ( $\alpha$ Cygni) we know $T=10,000^{\circ} \mathrm{K}$. Then

$$
\log \frac{n_{s}}{n_{s}{ }^{\prime}}+\log \frac{n_{t}^{\prime}}{n_{t}}=5040\left(E_{s}-E_{t}\right)\left(1 / T^{\prime}-1 / T\right) .
$$

The left-hand side is the observed ratio of the intensities. The result is

$$
T^{\prime}=4500^{\circ} \mathrm{K} \text {. }
$$

This is too high a value for the cool star and too low a value for the hot star. We conclude that the excitation is probably not produced by the continuous radiation of either star.

We have as yet no information concerning the location of the tenuous gas which emits the lines of [FeII] and FeII. In both, Boss 1985 and $W Y$ Geminorum, the components of their binary systems are unresolved on the slit of the spectrograph.

Nature was kind in this instance and provided us with one relatively near-by star in which similar bright lines of $[\mathrm{FeII}]$ are observed. This star is $\alpha$ Scorpii (Antares). It is a visual double star, whose principal component has a normal spectrum corresponding to a star of low temperature. The fainter component has a temperature of about $20,000^{\circ} \mathrm{K}$. In the spectrum of this hot star, there appear weak, narrow, emission lines of $[\mathrm{FeII}]$. When the image of the star is held stationary on the slit, the emission lines are seen to extend slightly on both sides of the normal continuous spectrum. We conclude:

(a) The emission of $[\mathrm{FeII}]$ occurs in the vicinity of the hot star and does not exist at other points located at a similar distance from the cool star; we do not, however, exclude the possibility of weak emission in the immediate vicinity of the cool star, because the latter is so bright that the plate is burned out in a few seconds.

(b) The extensions of the emission lines suggest that the hot star is surrounded by a nebulous shell or ring whose radius is of approximately the same size as the distance between the two stars.

(c) The absence of emission lines of [FeII] in other single stars having temperatures of $20,000^{\circ} \mathrm{K}$ shows that the nebulosity owes its origin to the binary character of Antares.

(d) The hot component of Antares is underluminous, thereby resembling the hypothetical hot component of $Z$ Andromedae.

We next turn to the question of the source of excitation. Two sources suggest themselves: (a) continuous radiation from the hot star in a nebulosity whose origin may be somewhat similar to that discussed by Kuiper in the system of $\beta$ Lyrae and (b) inelastic collisions with free electrons. Let us consider the first mechanism.

We have essentially three levels of $\mathrm{Fe}^{+}$, the ground level, a low metastable level, and a slightly higher normal level. Let the density of the exciting radiation be

$$
\Delta_{i k}=\beta \Delta_{\mathrm{Planck}}
$$


where $\beta \leqslant 1$ is the dilution factor. Then we find,

$$
\begin{gathered}
\text { when } \rho_{i k}=\frac{g_{i}}{g_{k}} \exp \left[-h \nu_{i k} / k T\right], \\
n_{3} / n_{1}=\beta \rho_{13}, \\
\frac{n_{2}}{n_{1}}=\rho_{12} \quad \text { if } \quad \beta \gg \frac{A_{21}}{A_{31}} ; \quad A_{21} \ll A_{31} \\
\frac{n_{2}}{n_{1}}=\beta \frac{A_{31}}{2 A_{21}} \text { if } \quad \beta \ll \frac{A_{2}}{A_{31}} ; \quad A_{21} \ll A_{31} .
\end{gathered}
$$

The relative intensities of permitted and forbidden lines are then given by the two limiting expressions $^{3}$

$$
\begin{aligned}
& \frac{\text { Int. (permitted) }}{\text { Int. (forbidden) }}=\frac{n_{3} A_{31}}{n_{2} A_{21}}=\beta \frac{\rho_{13} A_{31}}{\rho_{12} A_{21}}, \\
& \frac{\text { Int. (permitted) }}{\text { Int. (forbidden) }}=\frac{n_{3} A_{31}}{n_{2} A_{21}}=2 .
\end{aligned}
$$

Let $A_{31} / A_{21} \sim 10^{6} ; \rho_{13} / \rho_{12}=10^{-1}$. We find that (a) Int. (perm.)/Int. (forb.) is never less than one, and (b) Int. (perm.)/Int. (forb.) $\gg 1$ if $\beta>10^{-6}$. But from geometrical considerations $\beta=R^{2} / 4 r^{2}$ where $R$ is the radius of the star and $r$ is the distance of the excited nebulous mass from the center of the star. The condition $\beta>10^{-6}$ corresponds to $r / R<500$. In our example (Antares) $R \sim 7 \times 10^{10} \mathrm{~cm}$. The radius of the emitting shell is about 3 seconds of arc, and the distance of Antares is about 100 parsecs, or $3 \times 10^{20} \mathrm{~cm}$. Hence, $r \sim 5 \times 10^{15} \mathrm{~cm}$ and our condition for Int. (perm.)/Int. (forb.) 1 is certainly obeyed. But it is not apparent why the forbidden lines should be much stronger than the permitted lines, if the mechanism is that of radiative excitation from the hot star. It is true that we have neglected any possible departures from blackbody radiation in the exciting star, and that we have greatly simplified the problem considering only three states.

We next consider the problem of collisional excitation. The numbers of collisions from state 1 -we again limit ourselves to three states-are

$$
n_{1} n_{e} b_{12} \text { and } n_{1} n_{e} b_{13}
$$

where

$$
\begin{aligned}
& b_{i k}=\stackrel{2}{\sigma_{i k}} 2\left\{\frac{2 \pi k T}{m}\right\}^{\frac{1}{2}} e^{-\eta / k T}, \\
& b_{k i}=\stackrel{2}{2} 2\left\{\frac{2 \pi k T}{m}\right\}_{k i}^{\frac{1}{2}} .
\end{aligned}
$$

Very little is known about the cross sections $\sigma^{2}$. We shall assume $10^{-16} \mathrm{~cm}^{2}$. We next have approximately

$$
2\left\{\frac{2 \pi k T}{m}\right\}^{\frac{1}{2}} \approx 10^{8}, \quad e^{-\eta / k T} \approx 1, \quad b_{12} \approx 10^{-8} .
$$

We shall have predominantly collisional excitation if

or when

$$
n_{1} n_{e} b_{12} \gg n_{1} \Delta_{12} B_{12}
$$

$$
n_{e}>10^{15} \text { for } \beta=1 \text {. }
$$

This is slightly in excess of the electron density in the sun's reversing layer (Table I). If $\beta<1$, but $n_{e} / \beta=$ const, then we retain the same margin of safety. That is, for all shells in which the density decreases at least as rapidly as the dilution factor $\left(\sim 1 / r^{2}\right)$, the radiative processes of excitation will probably predominate. For example, in an expanding atmosphere with $v_{\exp }=$ const we have

$$
n_{e} \sim 1 / r^{2}, \quad \beta \sim 1 / r^{2}, \quad n_{e} / \beta=\text { const. }
$$

Hence, collisional processes should remain relatively unimportant in such shells as those of Pleione, 48 Librae, etc. But if the density decreases less rapidly than $1 / r^{2}$, then collisions may become more important. It is almost certain that in the peculiar double star systems of Antares, CI Cygni, etc., we are concerned with a nebulous mass whose density is greater than one produced by a uniformly expanding shell like $P$ Cygni. In fact, the state of ionization may serve as a useful criterion to decide about the density. If the normal reversing layer of the exciting star shows HeI, and other elements of high ionization, and the nebulous shell shows [FeII], we can conclude that the latter has a lower level of ionization than the reversing layer. Now, it is probable that the ionization is of photoelectric origin. Hence it is reduced by the 
same factor $\beta$. Lowered ionization should result from densities in excess of $1 / r^{2}$.

If the excitation of [FeII] and FeII is caused by collisions, then the peculiarly low fictitious excitation temperature takes on a very different meaning. To make further progress we need reliable determinations of the collisional probabilities $b_{i k}$.

\section{THE HeI PROBLEM}

A somewhat similar problem to that of the [FeII] lines arises when we consider the intensities of the HeI singlets and triplets in peculiar stellar spectra (Fig. 11). In the laboratory, as in almost all astronomical sources, the triplets are relatively stronger than the singlets. The "classical" theory accounts for this in a very satisfactory manner because of the larger statistical weights of the triplet levels. In normal stellar spectra, the intensities are affected by unrelated phenomena, such as the Stark effect and the combination of turbulent broadening with broadening caused by radiation damping. But these phenomena are now fairly well understood and there is nothing unexpected in the relative intensities. But when we consider the emission lines in a star like $R W$ Hydrae (Fig. 12) and compare them to the laboratory lines of HeI or to the lines of ordinary gaseous nebulae, we are struck by the fact that the members of the $2^{1} P-n^{1} D$ series are relatively strong as compared to the members of the $2^{3} P-n^{3} D$ series.

This phenomenon is of considerable theoretical interest. Some years ago K. Wurm and the writer computed the populations of the five lowest levels of $\mathrm{HeI}$ for two temperatures $\left(10,000^{\circ}\right.$ and $25,000^{\circ}$ ) and for dilution factors of $0.1,0.02$, and 0.01 . It was shown that in the case of dilute blackbody radiation the metastable levels gain in population relative to the non-metastable levels, as the dilution factor becomes smaller. Simultaneously, the triplet levels gain over the singlet levels, so that, for example, in an extended shell, like that of $\gamma$ Cassiopeiae as observed in 1940 , the lines $2^{3} P-n^{3} D$ were very much stronger than the lines $2^{1} P-n^{1} D$, and the lines $2^{3} S-n^{3} P$, $2^{1} S-n^{1} P$ dominated the entire spectrum. These computations were extended recently by $\mathrm{L}$.

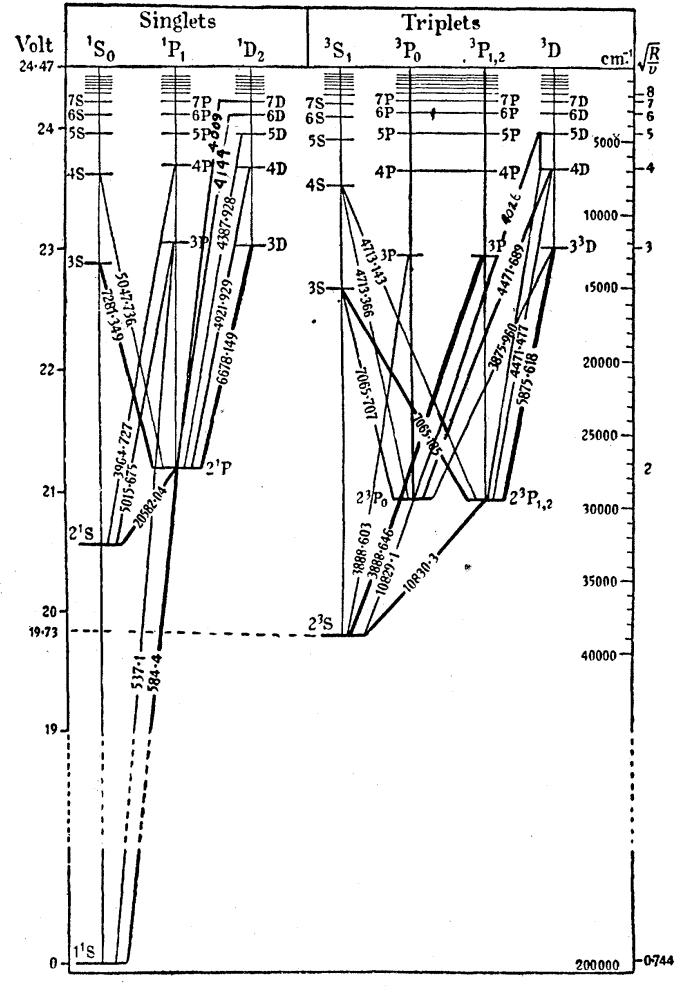

FIG. 11. Level diagram of helium.

Goldberg $^{8}$ for temperatures of $50,000^{\circ}$ and $100,000^{\circ}$, and for dilution factors which are much smaller than $10^{-3}$. The result is that "the influence of diluted radiation in overpopulating the metastable levels and, indeed, all triplet levels with respect to the singlets should be very much more pronounced in the nebulae than in the distended stellar atmospheres treated by Struve and Wurm." Thus, at $T=50,000^{\circ}$, Goldberg finds that the $2^{3} P$ level is about 88 times more populated than would be the case in thermodynamic equilibrium, while for the $2^{1} P$ level, the factor is almost exactly one.

Goldberg also showed that the higher triplet levels are overpopulated by a factor of about two as compared to the higher singlet levels. This seems to be in accordance with the observed fact that in the gaseous nebulae, the triplets in emission are very strong as compared to the singlets. But the latter are by no means completely suppressed, as one might have thought

${ }^{8}$ Astrophys. J. 93, 249 (1941). 
at first glance. In fact, it appears that very high exciting temperatures, rendering the mechanisms of ionization and recombination more important than the mechanism of line absorption, would gradually tend to establish a balance between triplets and singlets at some such value as the factor two found by Goldberg.

Since the mechanism of radiative excitation which we have discussed continuously favors the triplets, it is extremely puzzling that in some peculiar stars the singlets appear distinctly stronger than the triplets. The presence in emission of HeI, HeII, OIII, [OIII], [NeIII], etc., all of which require high ionization energies, and some of which, in addition, require high excitation energies, shows that the source of the emission must be a tenuous layer of gas subjected to intense excitation, under conditions favoring the production of forbidden lines, namely, very low density and great departure from thermodynamic equilibrium.

Let us, for convenience, simplify the problem and consider only four states ${ }^{3}$ the ground level of HeI, a composite level containing all excited singlets, a composite level containing all excited triplets, and the continuum. Also, let us first suppose that radiative processes alone are im- portant. Next, let us assume that

$$
\begin{array}{ll}
A_{24}=A_{34}, & \rho_{i k}=\exp \left(-h \nu_{i k} / k T\right), \\
A_{42}=A_{43}, & \rho_{14}=\rho_{12} \rho_{24}, \\
A_{42}=A_{41}, & \rho_{24}=\rho_{34} . \\
A_{21}=y A_{41}, &
\end{array}
$$

Let the dilution factor $\beta$ apply to transitions $1 \rightarrow 4 ; 2 \rightarrow 4 ; 3 \rightarrow 4$; but let another dilution factor, $\gamma$, apply to transition $1 \rightarrow 2$. The reason for making this distinction is that in the nebulosity, the energy density corresponding to the frequencies of the strong ultimate emission lines $1^{1} S-n^{1} P$ must be very great and is not simply that given by $\beta \Delta_{\text {Planck. }}$. A similar problem, in the case of hydrogen, leads to large energy densities in $L_{\alpha}$, and this is the basis of all modern theories of hydrogen nebulae. We shall have

$$
\gamma \gg \beta .
$$

Then

$$
\frac{\text { Singlets }}{\text { Triplets }}=n_{2} / n_{3}=\frac{2 \gamma y+\beta \rho_{24}}{y+\gamma y+\beta \rho_{24}} \approx 2 .
$$

The method is crude and only goes to show that we can enhance the singlets until they are ap-

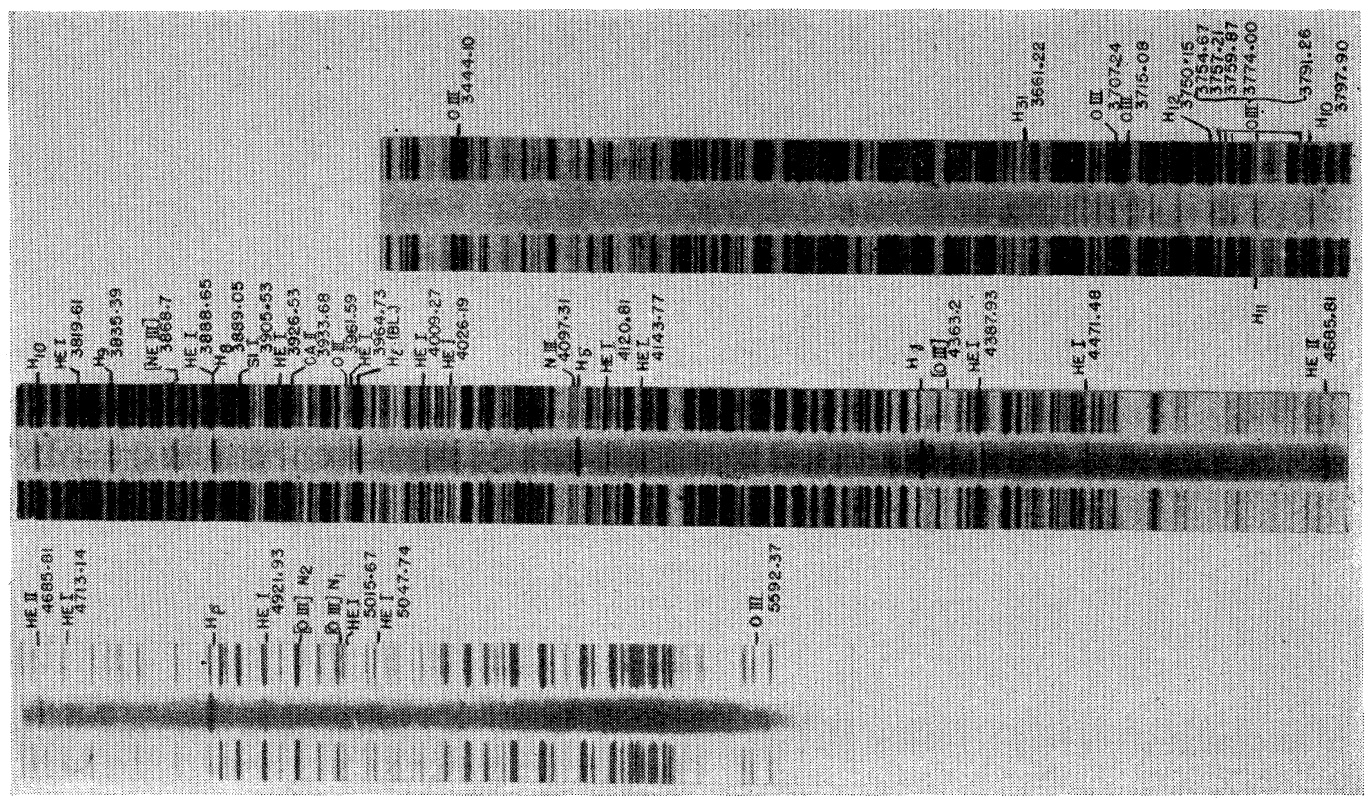

FIG. 12. $R W$ Hydrae. 
proximately equal to the triplets by the assumption $\gamma \gg \beta$. But it is not yet clear why this condition should not also apply in the case of the ordinary gaseous nebulae. If we have

$$
\gamma=\beta \ll 1, \quad n_{2} / n_{3} \approx \beta\left(2+\rho_{24}\right) \approx \beta .
$$

This is the case we discussed before, which shows that uniform dilution tends to build up the excited triplets at the expense of the excited singlets.

If it were possible, somehow, to avoid ionization completely then we should in principle be able to build up the singlets without exciting the triplets at all (if collisions are negligible). But it is difficult to see how these conditions could be realized when the exciting radiation comes from a star. As soon, however, as ionization sets in, atoms begin to be diverted into the triplet system where they remain, except through new ionizations and recombinations.

The question arises whether collisions from the ground state, or between excited levels, can account for the strengthening of the HeI singlets. But here, again, we are confronted by the lack of information - theoretical or experimental-of the collisional cross sections. It is probable that for collisions from the ground state to be important we must have

$$
n_{e} \gg 10^{18} \text { when } \beta=1
$$

and that collisions between excited levels may become significant when

$$
n_{e} \gg 10^{15} \text { for } \beta=1 \text {. }
$$

There have, of course, been many laboratory experiments to determine the collisional excitation functions for HeI. Some of them have shown a remarkable enhancement of the singlets over the triplets. It is extremely tempting to attribute the astronomical results to the same cause. But the theoretical interpretation of these excitation functions seems to be inadequate at the present time for any direct application. 


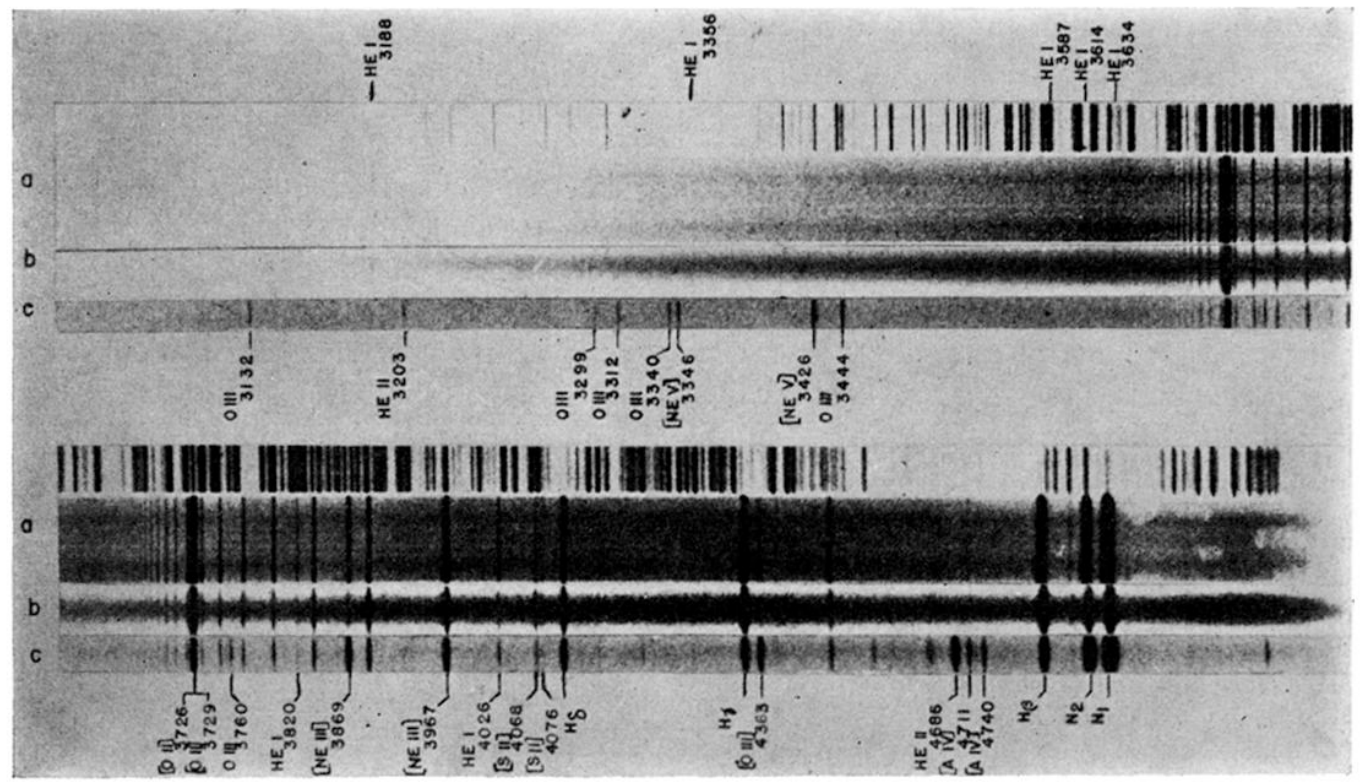

FIG. 1. Nebulae: (a) Orion, (b) IC 418, (c) IC 2165. 


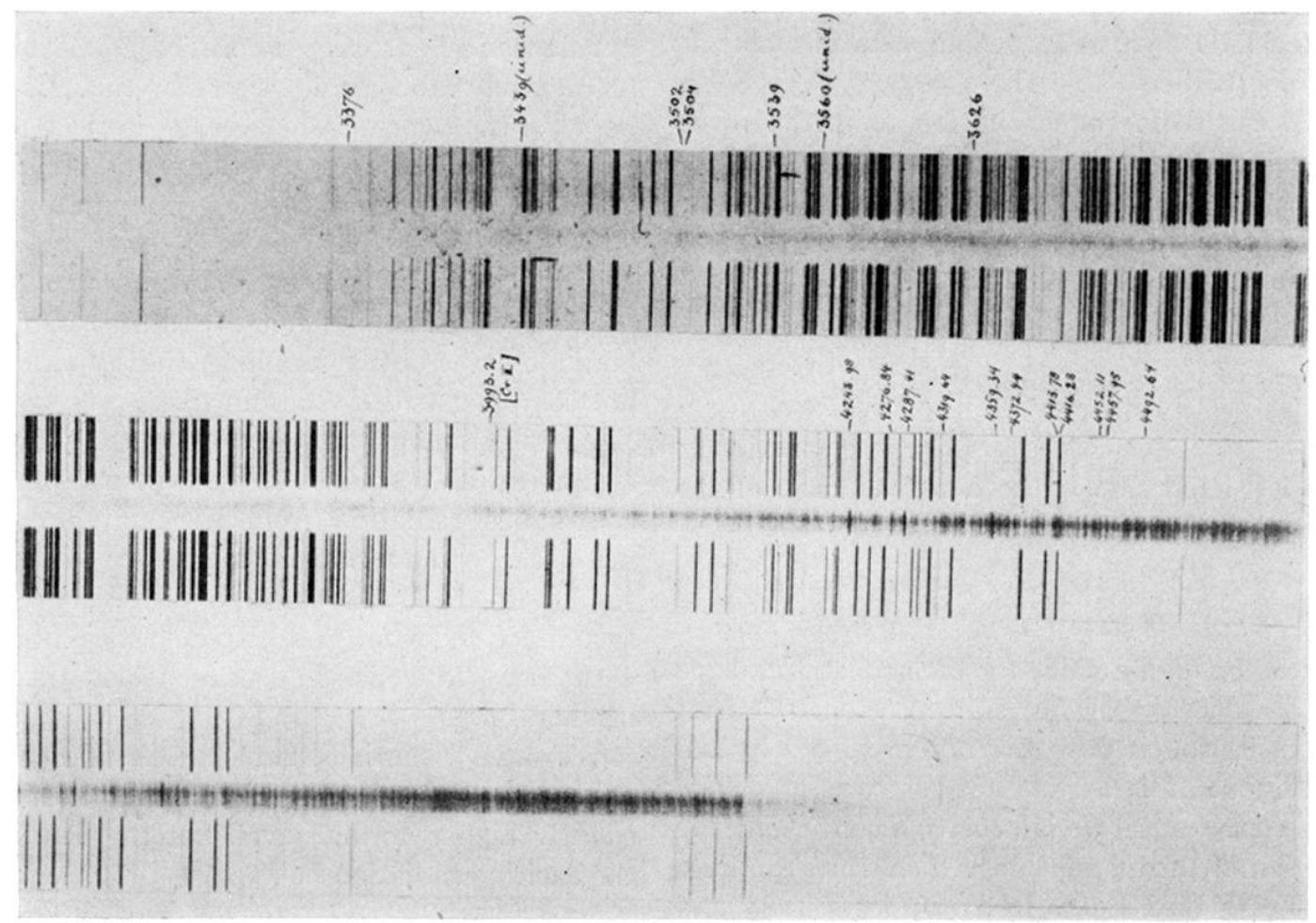

FIg. 10. $W Y$ Geminorum. 


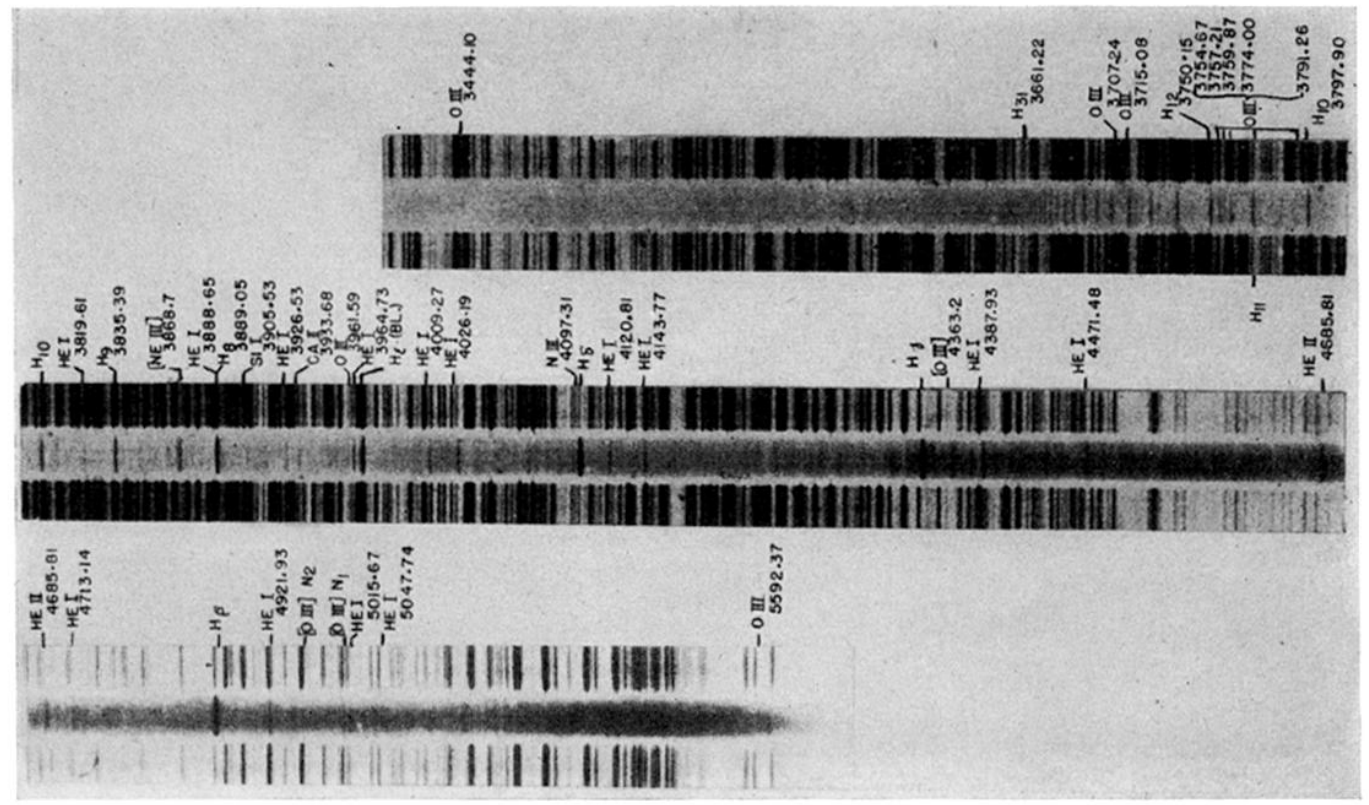

FIg. 12. $R W$ Hydrae. 


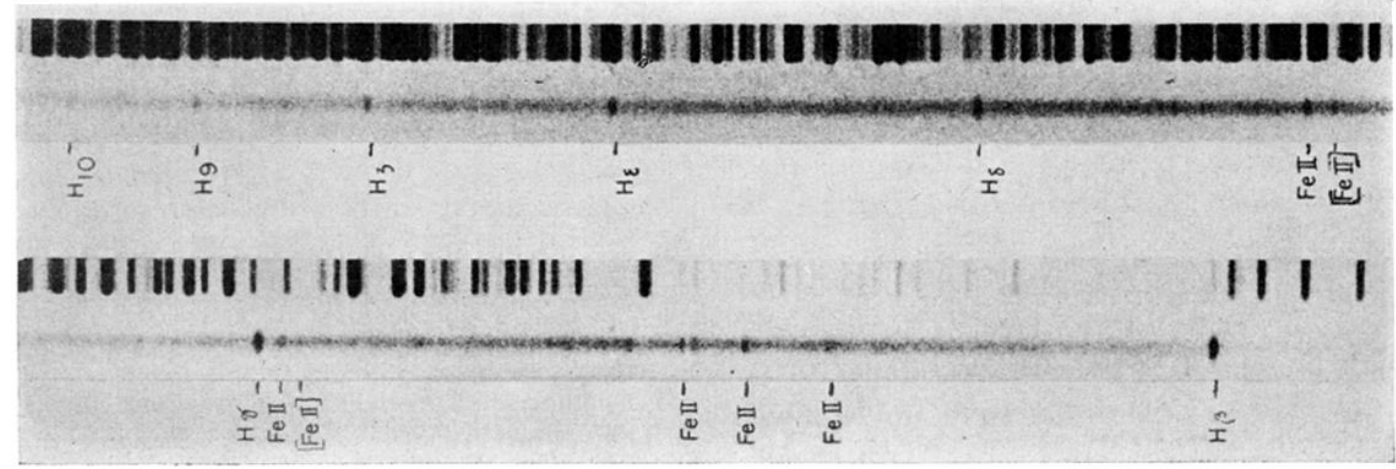

Fig. 2. $M W C 56$. 


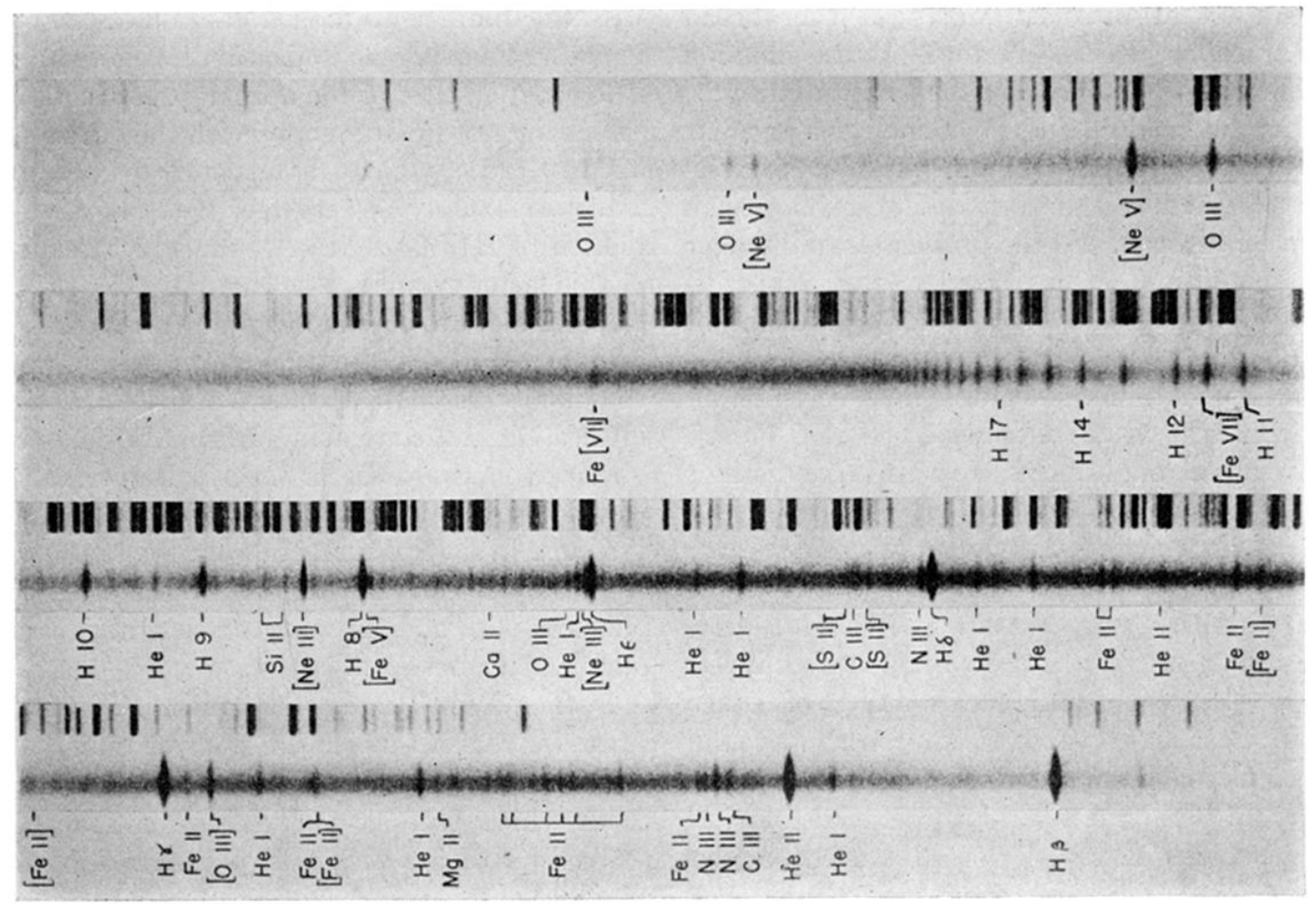

Fig. 3. $Z$ Andromedae. 


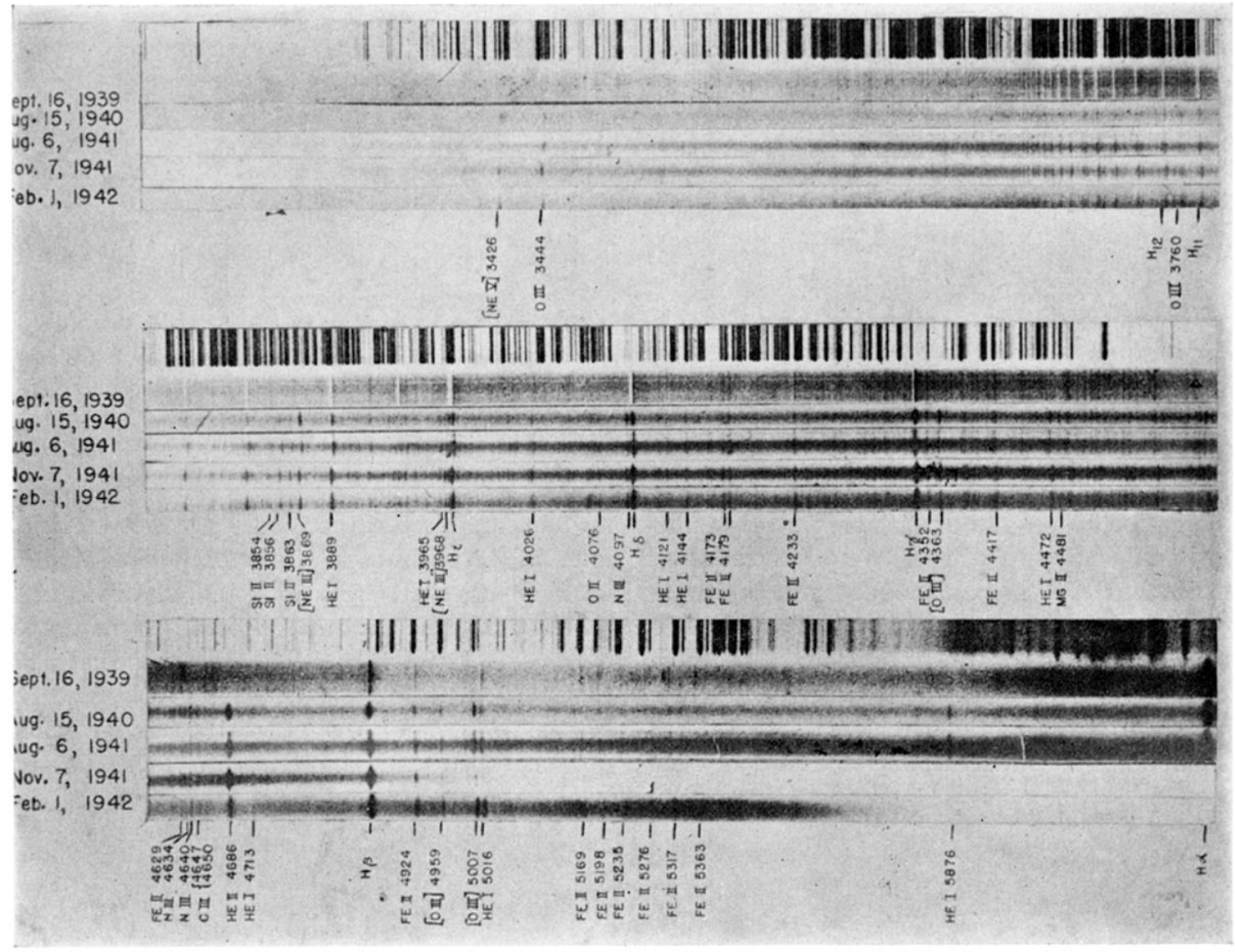

FIg. 4. Changes in the spectrum of $Z$ Andromedae. 


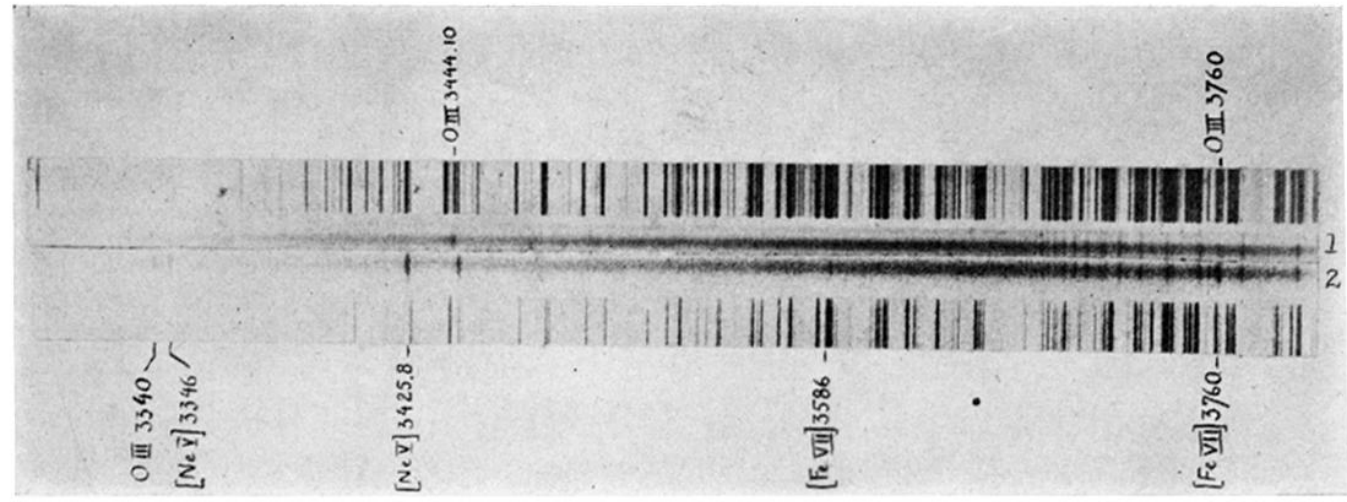

Fig. 5. $Z$ Andromedae. 


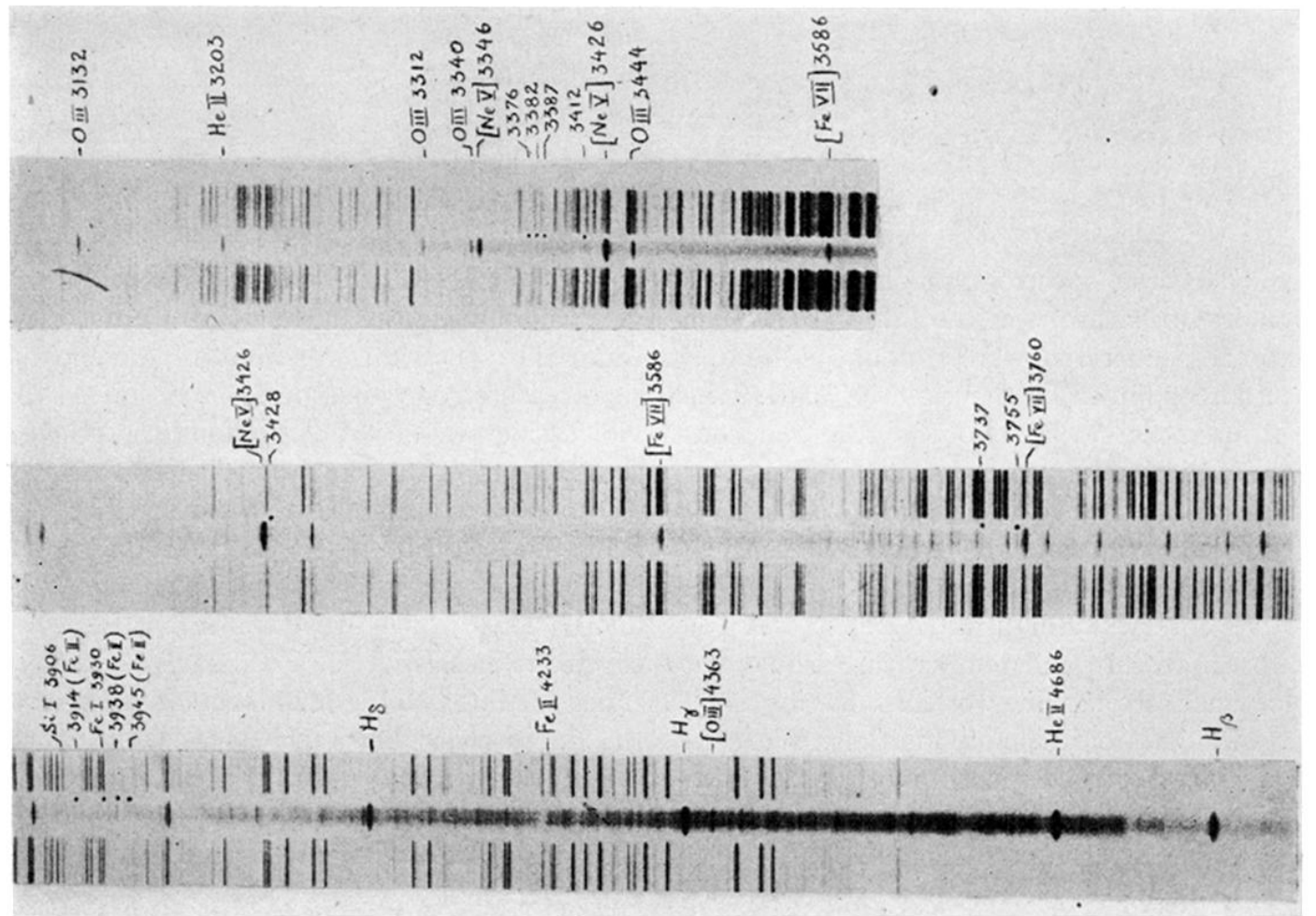

FIG. 6. CI Cygni. 


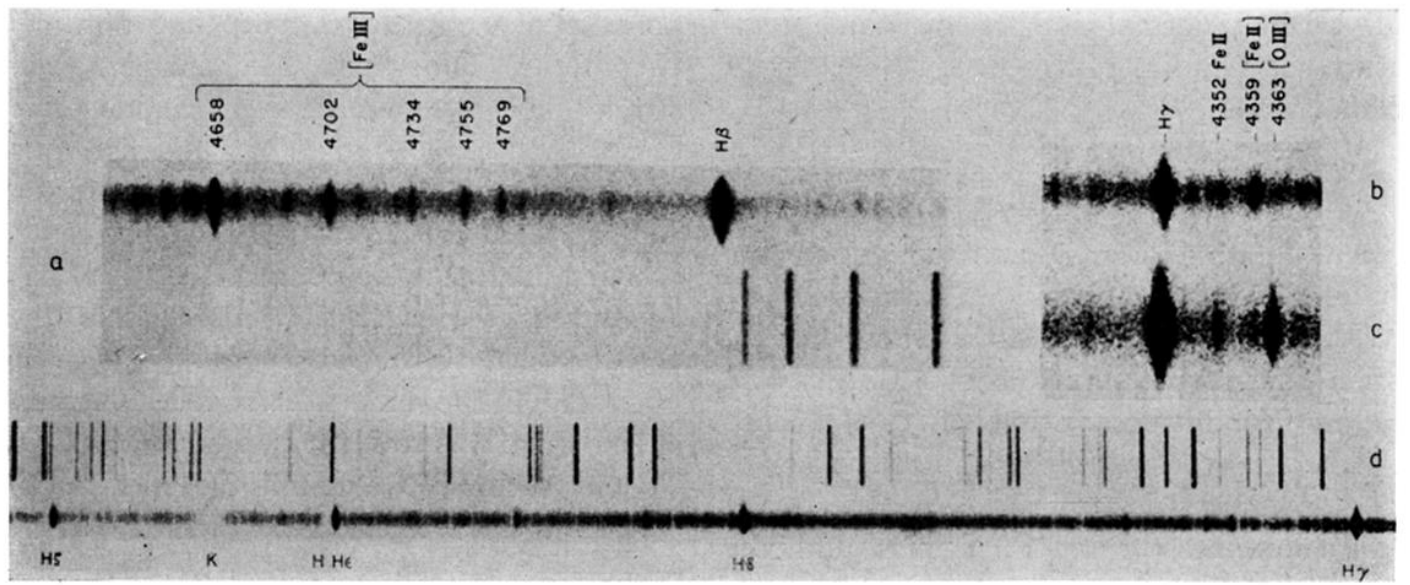

FIG. 7. BF Cygni (a) August 31, 1941; (b) August 6, 1941; (c) September 15, 1942; (d) August 11, 1943. 


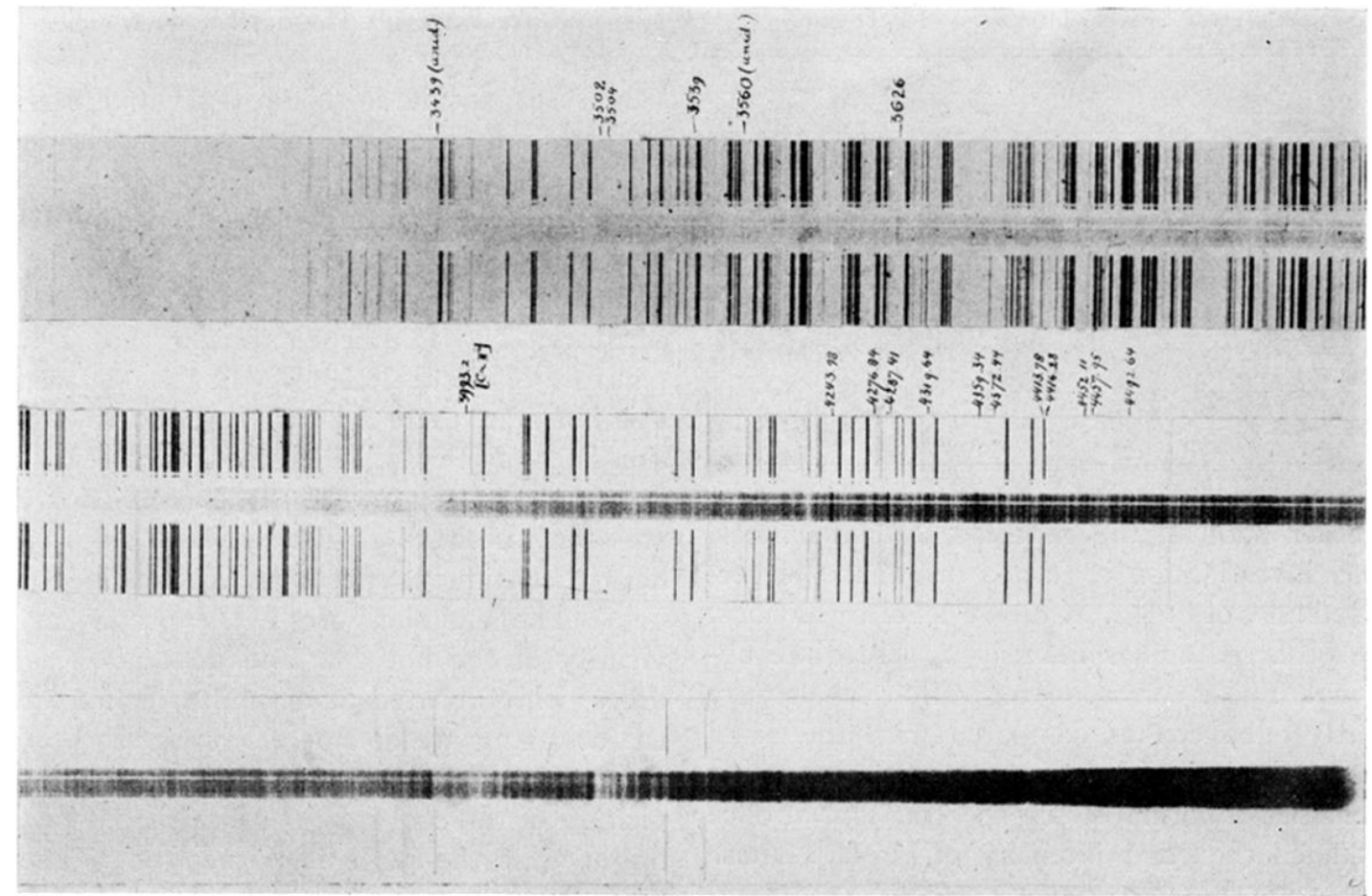

FIG. 9. Boss 1985. 